\title{
Unveiling the broad band X-ray continuum and iron line complex in Mrk 841
}

\author{
P. O. Petrucci ${ }^{1}$, G. Ponti ${ }^{2,3}$, G. Matt ${ }^{4}$, A. L. Longinotti ${ }^{5}$, J. Malzac $^{6}$, M. Mouchet ${ }^{7,8}$, \\ C. Boisson ${ }^{7}$, L. Maraschi ${ }^{9}$, K. Nandra $^{10}$, and P. Ferrando ${ }^{8,11}$
}

\author{
${ }^{1}$ Laboratoire d'Astrophysique de Grenoble, BP 43, 38041 Grenoble Cedex 9, France \\ e-mail: petrucci@obs.ujf-grenoble.fr \\ 2 Dipartimento de Astronomia, Università degli Studi di Bologna, via Zamboni, 40127 Bologna, Italy \\ 3 INAF-IASF Sezione di Bologna, via Gobetti 101, 40129 Bologna, Italy \\ 4 Dipartimento di Fisica, Università degli Studi "Roma tre", via della Vasca Navale 84, 00046 Roma, Italy \\ 5 XMM-Newton Science Operations Center, European Space Astronomy Center, ESA, 28080 Madrid, Spain \\ 6 Centre d'étude Spatiale des Rayonnements (CNRS/UPS/OMP), 31028 Toulouse, France \\ 7 LUTH, Observatoire de Paris, Section de Meudon, 92195 Meudon Cedex, France \\ 8 APC Universite Paris 7 Denis Diderot, 75205 Paris Cedex 13, France \\ 9 Osservatorio Astronomico di Brera, via Brera 28, 02121 Milano, Italy \\ 10 Astrophysics Group, Imperial College London, Blackett Laboratory, Prince Consort Road, London SW7 2AW \\ 11 Service d'Astrophysique, DSM/DAPNIA/SAp, CE Saclay, 91191 Gif-sur-Yvette Cedex, France
}

Received 4 May 2007 / Accepted 24 May 2007

\begin{abstract}
Context. Mrk 841 is a bright Seyfert 1 galaxy known to harbor a strong soft excess and a variable K $\alpha$ iron line. Historical UV, X and $\gamma$-rays observations show clear variation of its spectrum in flux and in shape on a monthly time scale.

Aims. Mrk 841 has been observed during 3 different periods (January 2001, January 2005 and July 2005) by XMM-Newton for a total cumulated exposure time of $\sim 108 \mathrm{ks}$. We present in this paper a broad band spectral analysis of the complete EPIC-pn data sets. These are the best observations so far for the study of the soft excess and iron line complex in this source.

Methods. We use different methods of data analysis including model-independent methods (spectral ratios, rms, ...) as well as model fitting. We were able to test two different models for the soft excess, a relativistically blurred photoionized reflection (REF model) and a relativistically smeared ionized absorption (ABS model). The continuum is modeled by a simple cut-off power law and we also add a neutral reflection.

Results. These observations confirm the presence of a soft excess and iron line and reveal extreme and puzzling spectral and temporal behaviors. The $0.5-3 \mathrm{keV}$ soft X-ray flux decreases by a factor 3 between 2001 and 2005 and the line shape appears to be a mixture of broad and narrow components, the former being variable on small $(\mathrm{ks})$ time scale while the later is consistent with being constant. The 2-10 keV spectrum also hardens between 2001 and 2005. We succeed in describing this complex broad-band $0.5-10 \mathrm{keV}$ spectral variability using either REF or ABS to fit the soft excess. Both models give statistically equivalent results even including simultaneous BeppoSAX data up to $200 \mathrm{keV}$. Both models are consistent with the presence of remote reflection characterized by a constant narrow component in the data. However they differ in the presence of a broad line component present in REF but not needed in ABS. Consequently the physical interpretation of the line profile variability is quite different, resulting from the variability of the broad line component in REF and from the variability of the absorbing medium in ABS. This study also reveals the sporadic presence of relativistically redshifted narrow iron lines, one of them being detected at $4.8 \mathrm{keV}$ in the EPIC-pn instruments at more than $98.5 \%$ confidence level. If interpreted as the blue horn of a relativistically distorted neutral iron line, the large redshift implies the presence of a Kerr black hole.
\end{abstract}

Key words. galaxies: Seyfert - galaxies: individual: Mrk 841 - X-ray: galaxies

\section{Introduction}

Seyfert 1 galaxies emit the bulk of their luminosity in the UV and X-ray bands. Their spectral energy distributions are characterized by two main components: a UV bump peaking in the UV range (O’Brien et al. 1988; Kinney et al. 1991), and an apparently non-thermal X-ray power law component extending up to at least $100 \mathrm{keV}$. A majority of objects show also the presence of a soft X-ray excess with respect to the high energy power law extrapolation (e.g. Walter \& Fink 1993; Page et al. 2004).

The origin of these components is not well understood. The basic paradigm supposes the existence of an accreting supermassive black hole. The gravitational energy released by the accreting gas is generally thought to be dissipated partly in the UV as thermal heating in an optically thick "cold" plasma (Shields 1978; Malkan \& Sargent 1982) and partly in X-rays in active blobs of optically thin "hot" plasma (e.g. Haardt et al. 1994). These blobs radiate mainly through comptonization of soft photons, possibly provided by the optically thick medium. Irradiation of the dense gas by hard X-rays produces also an X-ray reflection spectrum. The study of this reflected component (dominated by the iron $\mathrm{K} \alpha$ emission line and the reflection bump peaking between $20-40 \mathrm{keV}$ ) appears to be of great importance since it has the potential to be a key diagnostic of the strong gravity environment of black holes (Fabian et al. 2000, and references therein). 
Table 1. Observation epochs, total duration, $\%$ of good exposure time and mean count rates in the EPIC-pn instrument.

\begin{tabular}{lccccc}
\hline \hline & OBS 1 & OBS 2 & OBS 3 & OBS4 & OBS5 \\
\hline Start date & $2001-01-13$ & $2001-01-13$ & $2001-01-14$ & $2005-01-16$ & $2005-07-17$ \\
& $(05 \mathrm{~h} 20 \mathrm{~m} 55 \mathrm{~s} \mathrm{UT})$ & $(09 \mathrm{~h} 33 \mathrm{~m} 50 \mathrm{~s}$ UT $)$ & $(00 \mathrm{~h} 52 \mathrm{~m} 28 \mathrm{~s} \mathrm{UT})$ & $(12 \mathrm{~h} 38 \mathrm{~m} 21 \mathrm{~s} \mathrm{UT})$ & $(06 \mathrm{~h} 38 \mathrm{~m} 03 \mathrm{~s} \mathrm{UT})$ \\
Obs. ID & 0112910201 & 0070740101 & 0070740301 & 0205340201 & 0205340401 \\
Total duration (s) & 10106 & 12336 & 14775 & 49500 & 29509 \\
Good exp. time (\%) & 58 & 62 & 74 & 61 & 44 \\
Cts s $^{-1}$ & 17.9 & 22.2 & 21.8 & 5.6 & 7.2 \\
\hline
\end{tabular}

On the other hand, while the origin of the soft excess is still not clearly understood, it was recently realized that its characteristic temperature (when fitted by e.g. a simple black body) is remarkably constant over a wide range of AGN luminosities and black hole masses (e.g. Czerny et al. 2003; Gierliński \& Done 2004; Crummy et al. 2006; Ponti et al. 2006), favoring an origin through atomic processes instead of purely continuum emission. For example, recent studies suggest that an appealing explanation could be (photoionized) reflection from the accretion disc (Crummy et al. 2006). This spectral decomposition has been successful in fitting the XMM-Newton X-ray data of many sources like MCG-6-30-15, 1H 0707-495, NGC 4051 or MCG-02-14-009 (Fabian et al. 2004; Ponti et al. 2006; Larsson et al. 2007; Porquet 2006). Moreover, in those cases in which a broad Fe line is clearly detected (such as in MCG-6-30-15) the model is very attractive because the soft excess and broad Fe line are fitted self-consistently with the same relativistically blurred reflection model. But absorption instead of reflection could also reproduce the soft excess (Gierliński \& Done 2004, 2006; Sobolewska \& Done 2007; Schurch \& Done 2006) and modify the spectral shape close to the iron line to mimic the presence of a broad component. Nevertheless the reality is almost certainly a complex combination of absorption and reflection effects (e.g. Chevallier et al. 2006) and their relative importance in the observed spectra has been a significant topic of discussion in the recent literature. One of the main issues of this debate is the determination of the underlying continuum below the iron line in order to permit a precise measurement of the line broadness, the most important signature of the presence of black holes in AGNs.

Mrk 841 is a bright Seyfert 1 galaxy $(z=0.0365$, Falco et al. 1999), one of the rare Seyfert 1 s detected by OSSE at more than $3 \sigma$ (Johnson et al. 1997; Zdziarski et al. 2000). It is known for its large spectral variability (George et al. 1993; Nandra et al. 1995), its strong soft excess (this was the first object where a soft excess was observed, Arnaud et al. 1985) and its variable iron line (at least on a year time scale, George et al. 1993). The later was observed in some cases with a relatively large equivalent width (hereafter $E W$ ) of about $400 \mathrm{eV}$ (Day et al. 1990; Bianchi et al. 2001) significantly above the value predicted by standard cold reflection model (e.g. George \& Fabian 1991).

Recent XMM-Newton observations (Petrucci et al. 2002; Longinotti et al. 2004) have revealed a puzzling behavior of the iron line in Mrk 841. Indeed the presence of a highly variable, but narrow iron line feature was observed in XMM-Newton 2001, completely at odds with any currently-accepted interpretation of the line origin (Petrucci et al. 2002) and required further investigation. A re-analysis of the XMM-Newton data by Longinotti et al. (2004) proposed that the line may vary in width rather than in flux. Their interpretation then invokes local illumination by a flare inducing an hotspot in the inner disc, which then becomes progressively broadened as the disc rotates. On the other hand the reflection component, although poorly constrained due to the lack of high signal to noise in the simultaneous BeppoSAX data above $10 \mathrm{keV}$, was relatively large $(R>1)$ confirming a previous BeppoSAX observation (Bianchi et al. 2001). Astonishingly, the continuum shape and flux kept roughly constant between the two pointings as well as during the total (100 ks) BeppoSAX observation. For a better understanding of the puzzling spectral and temporal behavior of this source, it has been observed again in 2005 for a total duration of $\sim 75 \mathrm{ks}$.

We report here on the detailed spectral analysis of the whole XMM-Newton data set including one archive observation and the 4 open time pointings done in 2001 and 2005.

\section{The XMM-Newton data}

The first XMM-Newton (Jansen et al. 2001 and references therein) pointing of Mrk 841 (denoted OBS 1) was done on the 13th of January 2001 for $\sim 8 \mathrm{ks}$ as part of the guaranteed time program. It was immediately followed by the first open time observation. Due to operational contingency, the requested $30 \mathrm{ks}$ were split into two parts, on the 13th (OBS 2) and the 14th (OBS 3) of January 2001 with $\sim 11$ and 13 ks duration time respectively. The two observations were separated by about $15 \mathrm{~h}$. The source was re-observed 4 years later in January 2005. Here again the requested $75 \mathrm{ks}$ were split into two parts due to strong proton flares during the observation. About $46 \mathrm{ks}$ of good quality data were extracted from this pointing (OBS 4) while the $30 \mathrm{ks}$ left were performed in July 2005 (OBS 5). Table 1 gives a summary of the XMM-Newton pointings with the corresponding dates, observation duration and count rates.

In this paper we (usually) restrict the analysis to the EPIC-pn data. The EPIC-pn camera was always operated in Small Window mode, with thin aluminum filters to block visible light. The event files were reprocessed from the ODF data files using the epchain pipeline tasks of the XMM-Newton Science Analysis System (SAS version 6.5) and using the most updated version of the public calibration files. These event files were then filtered for good time intervals following the "recipe" given in the XMM-Newton SAS handbook (V2.01 23 July 2004, Sect. 5.2.4). Taking into account the dead time, the filtered event files then contained 5.9, 7.6, 9.4, 30.0 and $12.9 \mathrm{ks}$ of good exposure in the pn detector for OBS 1, 2, 3, 4 and 5 respectively. The final EPIC-pn count rates for each observation are reported in Table 1 . They are always well below the $1 \%$ pile-up threshold for both instruments. Due to the proximity of the "Small Window" edges, the source spectra and light curves were built from photons detected within a 40 arcsec extraction window centered on the source. X-ray events corresponding to pattern $\leq 4$ were selected. The background was estimated within a window of the same size as the source from an offset position.

In the following, all errors refer to $90 \%$ confidence level for 1 interesting parameter $\left(\Delta \chi^{2}=2.7\right)$. 


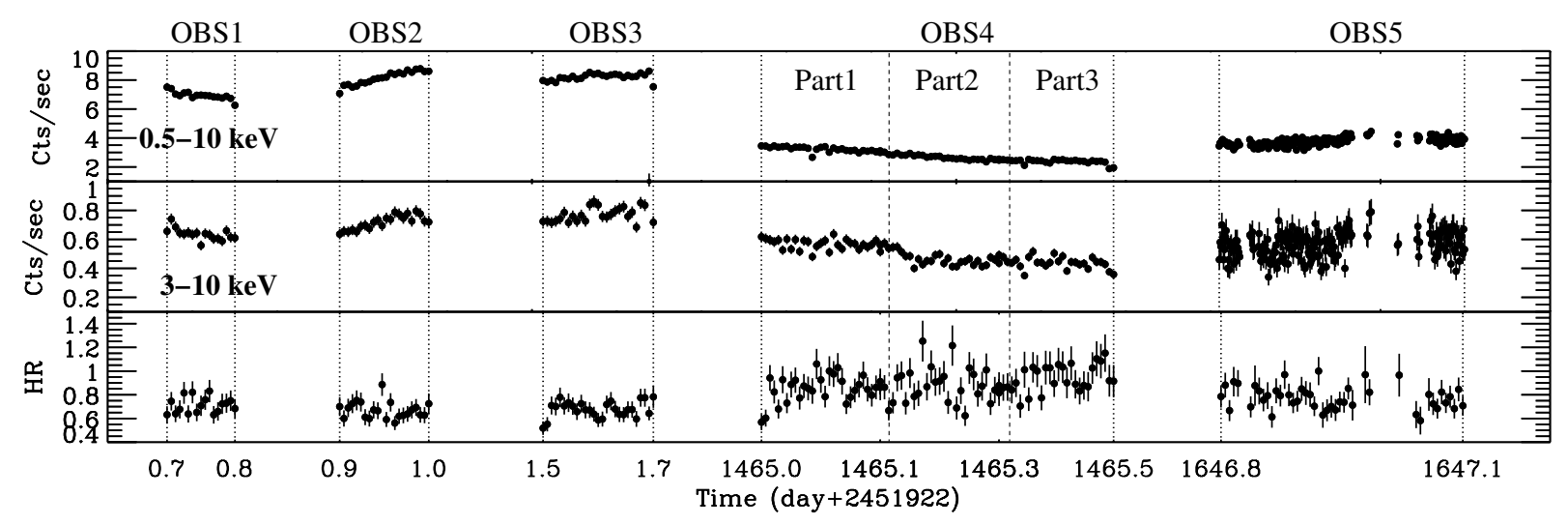

Fig. 1. Top: the $0.5-10 \mathrm{keV}$ X-ray light curve of the different EPIC-pn observations of Mrk 841 . It varies by a factor $~ 5$ in 4 years. Middle: the 3-10 keV X-ray light curve of the different EPIC-pn observations of Mrk 841. It varies by a factor two times lower than the 0.5-10 keV light curve meaning that the broad band variability is dominated by the soft $(<3 \mathrm{keV})$ band one. On the other hand, flux variability up to $\sim 50 \%$ is also observed on tens of ks in the soft and hard X-rays. Bottom: Hardness ratio light curve $(5-10 \mathrm{keV}) /(3-5 \mathrm{keV})$.

\section{Model-independent analysis}

\subsection{Light curves and hardness ratios}

We have plotted in the upper and middle panel of Fig. 1 the $0.5-10 \mathrm{keV}$ and 3-10 keV EPIC-pn count rate light curves of the different XMM-Newton observations of Mrk 841 as well as the hardness ratio $(5-10 \mathrm{keV}) /(3-5 \mathrm{keV})$ in the lower panel. The time binning is $500 \mathrm{~s}$. The total $0.5-10 \mathrm{keV}$ count rate decreases by a factor $\sim 5$ in 4 years while the hardness ratio increases, reaching maximum values during OBS 4. Between 2001 and 2005 , the $3-10 \mathrm{keV}$ count rate shows variations a factor two lower than the total count rate meaning that the broad band count rate variability is dominated by the soft $(<3 \mathrm{keV}) \mathrm{X}$-ray variability, at least on long time scale. Smooth soft and hard flux variabilities up to $\sim 50 \%$ are also visible on timescale of tens of ks.

\subsection{RMS}

Figure 2 shows the rms spectra of the different observations. The rms function has been calculated following the procedure detailed in Ponti et al. (2004). We use the 0.5-10 keV energy band. In order to increase the statistics we have grouped OBS 1, 2 and 3 to produce a single "2001" rms spectrum. The time binning is of about $1 \mathrm{ks}$ for each variability spectrum while the energy binning has been chosen in order to have negligible Poisson noise.

Due to the low degree of variability and the statistics we were not able to produce a spectrum with a large number of energy bins especially at high energy. Nevertheless the different rms spectra are relatively flat: OBS $1 / 2 / 3$ and OBS 5 are consistent with a constant value of $\sim 4$ and $5 \%$ respectively. On the other hand the rms spectrum of OBS 4 is inconsistent with a constant (at more than $99.99 \%$ ) with a mean value of $12 \%$. In this case we note a rough increase of the rms from 0.5 to about 2-3 keV and then a clear decreases at higher energies down to a few percent.

From now on, due to the flux and spectral variability observed in OBS 4, we divide this observation in 3 parts (noted part 1, part 2 and part 3 ) of about $15 \mathrm{ks}$ duration each as indicated in Fig. 1. Each part is analyzed separately.

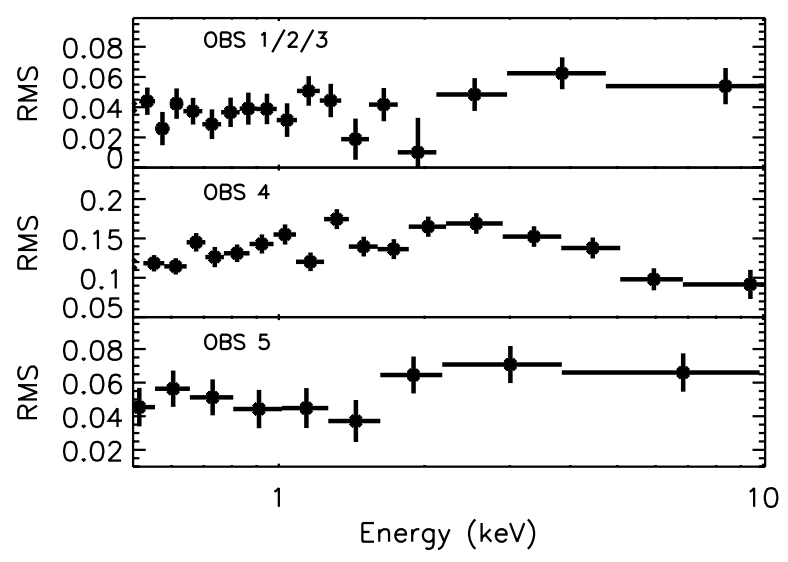

Fig. 2. rms spectra of OBS 1/2/3, OBS 4 and OBS 5.

\subsection{Spectral ratios}

We have plotted in Fig. 3 the ratios of the different EPIC-pn spectra with the EPIC-pn spectrum of OBS1. The binning is such that each bin has a $5 \sigma$ confidence level. No strong spectral variations are observed on short ( $\sim$ hours) time scale between OBS 1 , OBS 2 and OBS 3 or the three parts of OBS 4. However, the 3-8 keV spectral shape clearly hardens between 2001 and 2005 , in agreement with the increase of the hardness ratio shown in Fig. 1. Above $8 \mathrm{keV}$ the spectra keep roughly constant at least in shape. This spectral variability is more apparent in Fig. 4 where we have plotted the ratio between OBS 1 and the complete data set of OBS 4. For comparison, we have over-plotted in this figure the expected variability assuming two different cases: a power law pivoting around $8 \mathrm{keV}$ whose photon index decreases by 0.4 (dot-dashed line) and a variable black body peaking at $0.14 \mathrm{keV}$ and varying by a factor 3 in flux (dashed line). These values have been chosen just to illustrate what kind of variability we can expect in such cases. Clearly neither of these simulations is able to reproduce the complete spectral variability suggesting a more complex behavior. We can also notice in Fig. 3 some variations of the iron line complex on long (i.e. year like between OBS 1 and part 3 of OBS 4) and short (i.e. hours like between the different parts of OBS 4) time scale. 


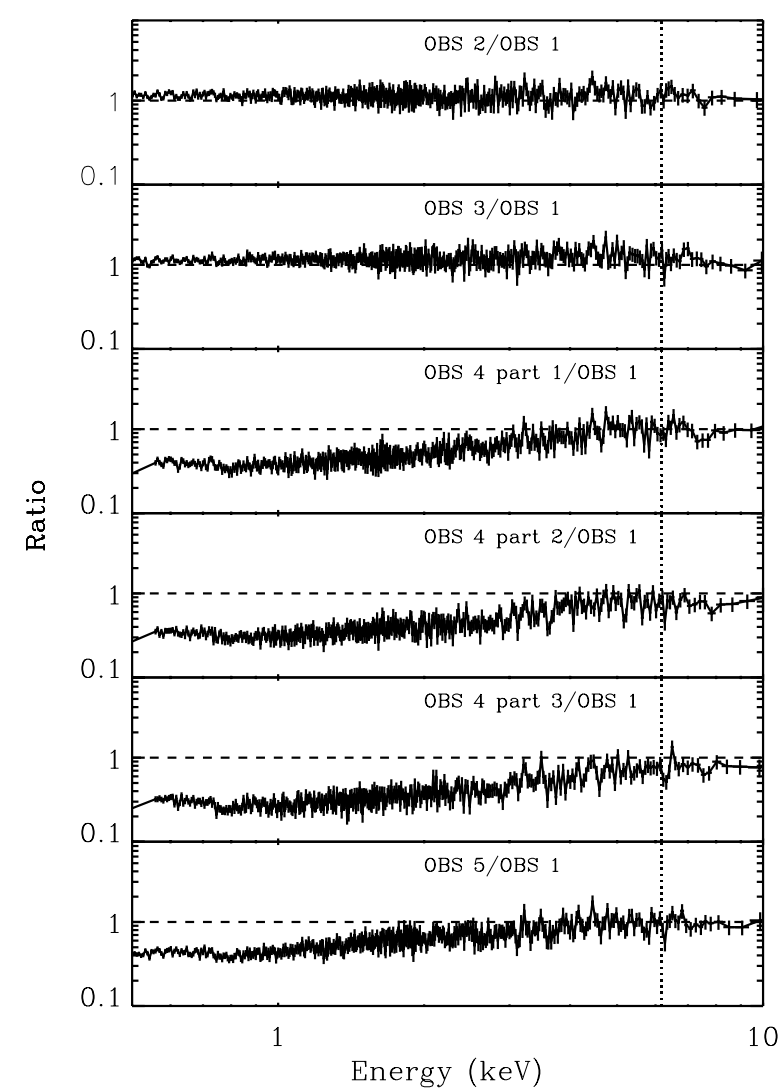

Fig. 3. Ratios between the different EPIC-pn spectra and the EPIC-pn spectrum of OBS1. The dashed line correspond to the position of the $6.4 \mathrm{keV}$ line in the source frame.

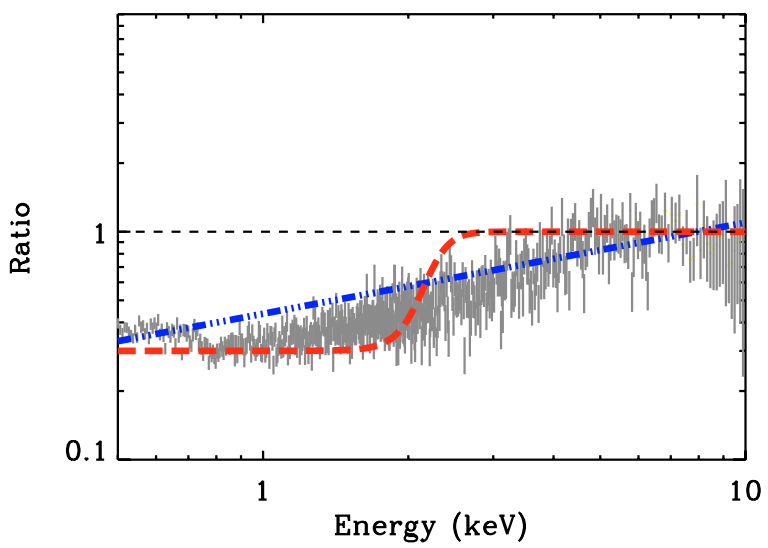

Fig. 4. Ratio between the EPIC-pn spectrum of the complete OBS 4 and the EPIC-pn spectrum of OBS1. The data (white grey lines) have been rebinned in order to have a $5 \sigma$ confidence level or at least 25 data points per bin. We have over-plotted in this figure, the expected variability in two different cases: a pivoting power law around $8 \mathrm{keV}$ whose photon index decreases by 0.4 (blue, dot-dashed line) and a variable black body peaking at $0.14 \mathrm{keV}$ and varying by a factor 3 in flux (red, dashed line).

\section{Spectral analysis}

\subsection{Phenomenological analysis}

Our first step in the spectral analysis was to fit the data with a simple power law, excluding the data below $3 \mathrm{keV}$. Figure 5 shows the ratio between the XMM-Newton/EPIC-pn data of part 1 of OBS 4 with the corresponding best fit power law. We

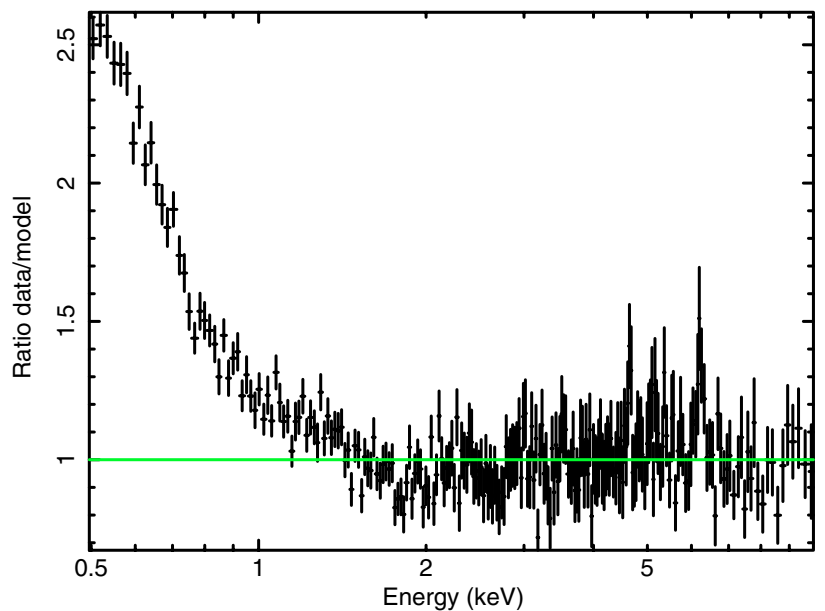

Fig. 5. Ratio data/model for part 1 of OBS 4. The model is a simple power law fitted between 3 and $10 \mathrm{keV}$ and extrapolated down to low energies. A strong soft excess and a line near $6 \mathrm{keV}$ are clearly apparent. Note also the presence of a narrow feature near $4.8 \mathrm{keV}$. It will be discuss in Sect. 5.

clearly observed a strong soft excess below $2 \mathrm{keV}$ and a fluorescent iron line complex near $6.4 \mathrm{keV}$.

The second step was to include very simple spectral components to reproduce the observed features. We use a power law for the continuum and a Gaussian for the iron line. A more precise analysis of the line complex is done in the next section. We fit the data above $3 \mathrm{keV}$ first. Then we fix the different parameters and include the data below $3 \mathrm{keV}$ down to $0.5 \mathrm{keV}$ and we add a simple multicolor accretion disc component (DISCBB in XSPEC) to model the soft excess. The best fit parameter values obtained with this method for the continuum, the multicolor disc and the line are reported in Table 2.

The best fits are clearly never satisfactory and large discrepancies are present especially below $3 \mathrm{keV}$ due to the bad black body approximation for the soft excess. Nevertheless several remarks, weakly affected by the goodness of the fit, can still been made:

- The photon index reaches values as small as 1.3 during OBS 4 where the flux is the lowest.

- The flux variation in the soft band $(<3 \mathrm{keV})$ between 2001 and Jan. 2005 is more than a factor 2 larger than the flux variation above $3 \mathrm{keV}$.

- The iron line width appears variable on relatively short time scale ( $<15 \mathrm{ks})$ e.g. between part 1 and part 2 of OBS 4.

The spectral variability agrees with the results shown previously with model-independent methods and suggest a spectral pivot at high energy. However the presence of a simple power law continuum is unlikely given the unusually (for a Seyfert galaxy) hard spectral index of OBS 4. More physical models are discussed in Sect. 4.3. Concerning the iron line, the 2005 observations confirm the apparent line variability already observed in 2001 (Petrucci et al. 2002; Longinotti et al. 2004). The best fits reported in Table 2 indicate the presence of a narrow line (compared to the EPIC-pn resolution) except OBS 3 and part 2 of OBS 4 where a broad component is preferred. The contour plots (at 68 and 90\% confidence level) of the line flux vs. line width for the different observations are plotted in Fig. 6 for 2001 (left plot) and 2005 (right plot). The line variability is discussed in more detail in the next section. 
Table 2. Best fit values obtained with a simple model including a power law for the continuum, a Gaussian for the iron line and a multicolor disc for the soft excess. The Gaussian energy is given in the source frame. The hydrogen column density, not shown in this table, is always consistent with the galactic one, i.e. $2.34 \times 10^{20} \mathrm{~cm}^{-2}$. The $\Delta \chi^{2}$ values correspond to the change in $\chi^{2}$ when adding the Gaussian component.

\begin{tabular}{lccccccccccc}
\hline \hline Obs & $\Gamma$ & $\begin{array}{c}E_{\mathrm{FeK}_{\alpha}} \\
\mathrm{keV}\end{array}$ & $\begin{array}{c}\sigma_{\mathrm{FeK}_{\alpha}} \\
\mathrm{eV}\end{array}$ & $\begin{array}{c}F_{\mathrm{FeK}_{\alpha}} \\
10^{-5}\end{array}$ & $\begin{array}{c}E W \\
\mathrm{eV}\end{array}$ & $\begin{array}{c}k T_{\mathrm{bb}} \\
\mathrm{keV}\end{array}$ & $N_{\mathrm{bb}}$ & $\begin{array}{c}F_{0.5-3} \\
10^{-11}\end{array}$ & $\begin{array}{c}F_{3-10} \\
10^{-11}\end{array}$ & $\Delta \chi^{2}$ & $\chi^{2} /$ d.o.f. \\
\hline 1 & $1.81_{-0.09}^{+0.07}$ & $6.25_{-0.14}^{+0.15}$ & $<550$ & $1.4_{-0.6}^{+3.2}$ & $90_{-35}^{+210}$ & $0.20_{-0.01}^{+0.01}$ & $547_{-60}^{+70}$ & 1.6 & 1. & 17 & $548 / 244$ \\
2 & $1.91_{-0.08}^{+0.06}$ & $6.39_{-0.05}^{+0.06}$ & $135_{-55}^{+65}$ & $2.7_{-0.8}^{+1.0}$ & $170_{-50}^{+60}$ & $0.18_{-0.01}^{+0.01}$ & $961_{-100}^{+110}$ & 1.9 & 1.1 & 37 & $510 / 263$ \\
3 & $1.95_{-0.12}^{+0.81}$ & $6.54_{-0.41}^{+0.40}$ & $950_{-570}^{+1020}$ & $5.3_{-2.0}^{+137.0}$ & $300_{-200}^{+8400}$ & $0.16_{-0.01}^{+0.01}$ & $1210_{-60}^{+70}$ & 1.9 & 1.3 & 32 & $397 / 263$ \\
4 part 1 & $1.43_{-0.07}^{+0.05}$ & $6.44_{-0.04}^{+0.05}$ & $<130$ & $1.3_{-0.5}^{+2.5}$ & $90_{-40}^{+170}$ & $0.13_{-0.01}^{+0.01}$ & $1996_{-359}^{+376}$ & 0.8 & 1.0 & 22 & $334 / 273$ \\
4 part 2 & $1.42_{-0.04}^{+0.09}$ & $5.50_{-0.43}^{+0.52}$ & $1200_{-600}^{+2300}$ & $5.9_{-4.1}^{+25.5}$ & $420_{-200}^{+1800}$ & $0.14_{-0.01}^{+0.01}$ & $1021_{-140}^{+200}$ & 0.6 & 0.8 & 14 & $342 / 266$ \\
4 part 3 & $1.30_{-0.06}^{+0.07}$ & $6.51_{-0.03}^{+0.03}$ & $<80$ & $1.6_{-0.4}^{+2.1}$ & $140_{-40}^{+80}$ & $0.14_{-0.01}^{+0.01}$ & $1008_{-130}^{+223}$ & 0.6 & 0.8 & 37 & $367 / 266$ \\
5 & $1.65_{-0.06}^{+0.05}$ & $6.49_{-0.07}^{+0.09}$ & $130_{-50}^{+110}$ & $1.9_{-0.8}^{+0.5}$ & $140_{-60}^{+40}$ & $0.12_{-0.01}^{+0.01}$ & $3348_{-680}^{+720}$ & 0.9 & 0.9 & 25 & $389 / 281$ \\
\hline
\end{tabular}
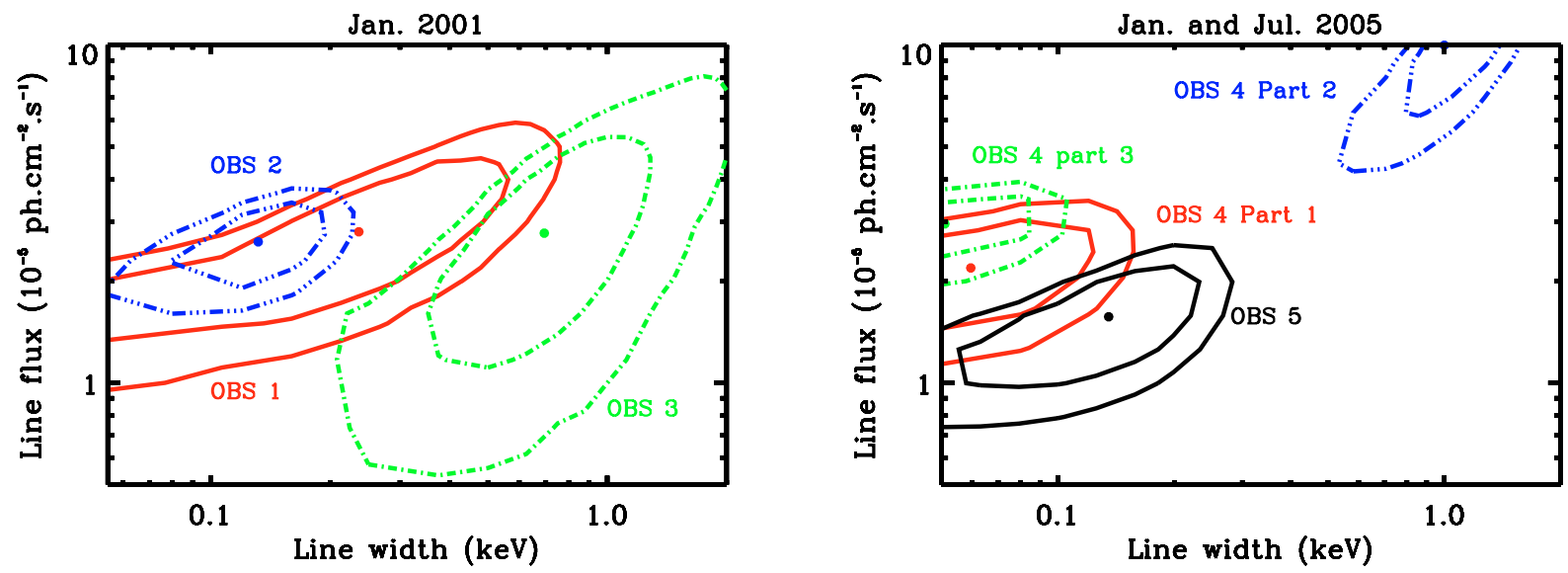

Fig. 6. Contour plots (68 and 90\%) of the line width vs. line flux obtained for the 3 observations of Jan. 2001 (left) and the 3 parts of Jan. 2005 as well as Jul. 2005 (right). The model includes a simple power law + Gaussian line.

To decrease the number of degrees of freedom, we choose to follow the fitting procedure of Longinotti et al. (2004). We fit together observations with roughly the same underlying continuum shape (cf. Table 2), e.g. the three pointings of 2001, on the one hand, and the different part OBS 4, on the other, keeping the power law continuum constant in shape but not in flux between the different spectra. OBS 5 is analyzed as a single observation.

\subsection{The iron line complex}

\subsubsection{Rapid variability}

The rapid ( $\sim$ hours time scale) variability of the line complex seems to be a common characteristic of this source. For a better visualization of this variability we have plotted in Fig. 7 the excess map of the complete set of the XMM-Newton/EPIC-pn observations. This map has been obtained following the method of Iwasawa et al. (2004) and Tombesi et al. (2007) applied for NGC 3516 and NGC 3783 respectively. We use resolutions of $2 \mathrm{ks}$ in time and $100 \mathrm{eV}$ in energy. Each temporal slice represents the residuals obtained when fitting each $2 \mathrm{ks}$ spectrum with a power law between 3.5 and $8 \mathrm{keV}$ but ignoring data between 4 and $7 \mathrm{keV}$. This map is useful to reveal narrow features but signatures of broad ones can be mixed up with the underlying continuum. A narrow feature close to $6.4 \mathrm{keV}$ is clearly present during most of the different pointings, but it seems to disappear from time to time on a very short time scale $(\sim \mathrm{ks})$ like in OBS 3 or in the middle of OBS 4 and OBS 5.

To check if the narrow line component variability is real or not we produce the light curves of its flux and equivalent width.
They are plotted in Fig. 8 with a 4 ks binning time scale. The model includes a power law and a narrow $(\sigma=0 \mathrm{eV})$ Gaussian line with energy fixed at $6.4 \mathrm{keV}$. We fit the data between 3 and $10 \mathrm{keV}$. From time to time the narrow line is poorly detected with only upper limit on its flux and $E W$. However both are consistent with a constant (at more than 98\%) from 2001 to 2005. The fact that the $E W$ is also constant is not surprising given the relative constancy of the underlying continuum near $6.4 \mathrm{keV}$ (see Fig. 3). A constant narrow line flux suggests the presence of remote reflection and it is discussed in the next section. If this interpretation is correct, the apparent variability shown by the excess map may reveal changes in either the continuum or any broad line emission underlying rather than changes of the narrow line itself. This is discussed in Sect. 4.2.3.

\subsubsection{Remote reflection?}

The presence of a roughly constant narrow line component, as shown in Fig. 8, suggests the presence of neutral reflection from remote material. Such reflection may come from the outer part of the accretion disc or even farther away from e.g. the dusty torus surrounding the nucleus as expected in the unification framework of AGNs.

In Table 3 we report the best fit parameters of the narrow $(\sigma=0 \mathrm{eV}$ ) Gaussian line added to the 3-10 keV power law best fit of the three observations of 2001 keeping the power law flux free to vary between OBS 1,2 and 3. We apply the same method for the three parts of OBS 4. OBS 5 is analyzed normally as a single observation. The contour plots of the narrow line flux vs. line energy are plotted in Fig. 9. The line fluxes 

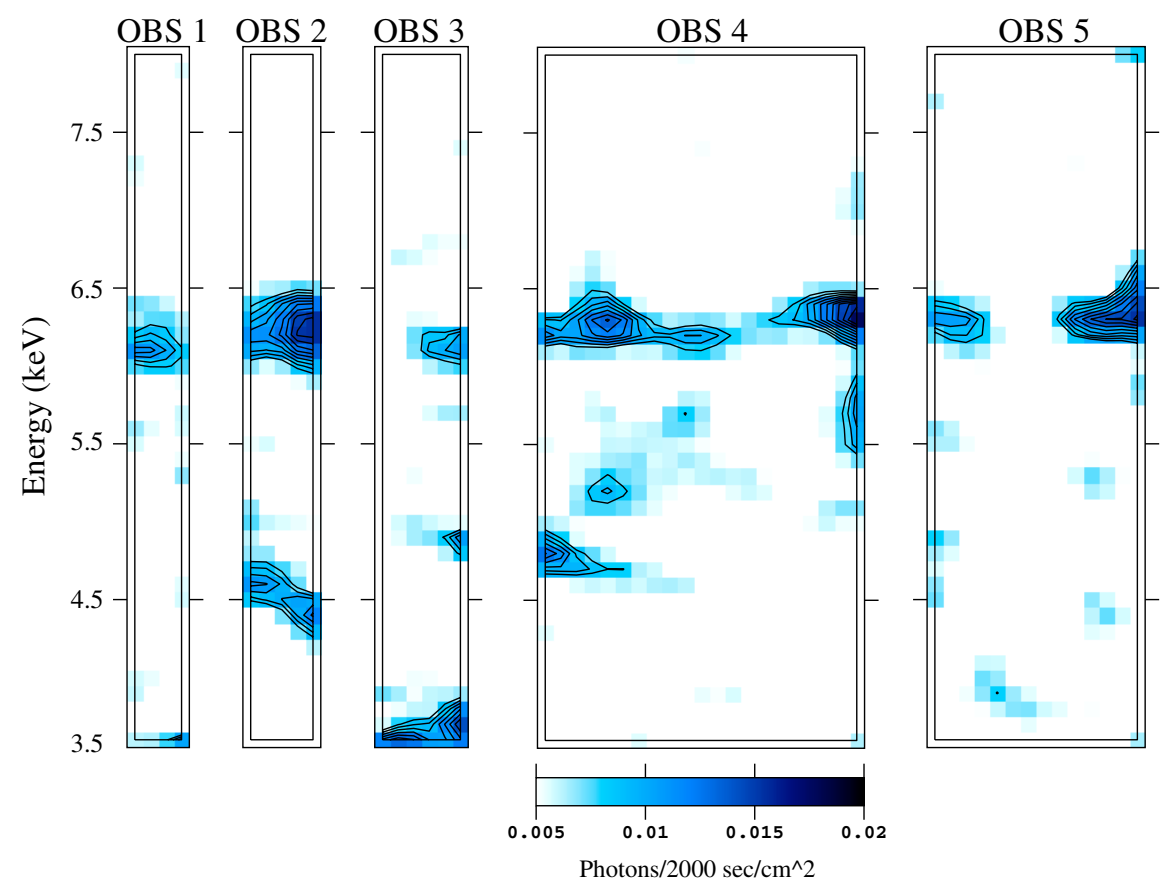

Fig. 7. Excess map on time-energy plane of the different XMM observations. The energy scale is in the lab frame.

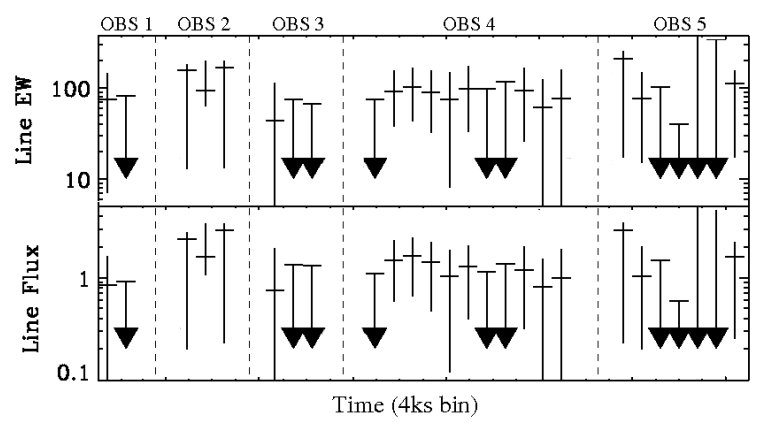

Fig. 8. Equivalent width (top, in eV) and flux (bottom, in $\left.10^{-5} \mathrm{ph} \mathrm{cm}^{-2} \mathrm{~s}^{-1}\right)$ light curves of a narrow $(\sigma=0 \mathrm{eV})$ Gaussian line whose energy is fixed to $6.4 \mathrm{keV}$. We use a $4 \mathrm{ks}$ time binning.

Table 3. Best fit parameters of the narrow $(\sigma=0 \mathrm{eV})$ Gaussian line added to mimic emission from remote material. The line energy is given

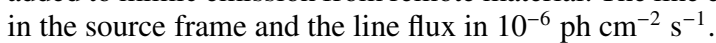

\begin{tabular}{lcc}
\hline \hline Obs & $E_{\mathrm{FeK}_{\alpha}}$ & $F_{\mathrm{FeK}_{\alpha}}$ \\
\hline 1/2/3 & $6.31_{-0.10}^{+0.05}$ & $10.2_{-3.1}^{+2.7}$ \\
4 part 1/2/3 & $6.45_{-0.02}^{+0.02}$ & $10.4_{-2.3}^{+2.3}$ \\
5 & $6.49_{-0.06}^{+0.04}$ & $10.2_{-4.5}^{+3.1}$ \\
\hline
\end{tabular}

obtained in the different observation periods are consistent with each other, in agreement with a roughly constant remote reflection. Surprisingly enough, while the best fit energy of the line is consistent with a slightly ionized iron line in 2005 (the $90 \%$ confidence level energy range being in between 6.43 and $6.47 \mathrm{keV}$ for OBS 4 and in between 6.43 and $6.54 \mathrm{keV}$ for OBS 5) it is smaller than and marginally consistent with $6.4 \mathrm{keV}$ (at less than $10 \%$ confidence level) in 2001.

In this last case, the detected line may be the signature of a slightly redshifted narrow iron line. This interpretation will be discussed in more detail in Sect. 5. If it is correct, the presence of remote reflection should still be tested for. We check the

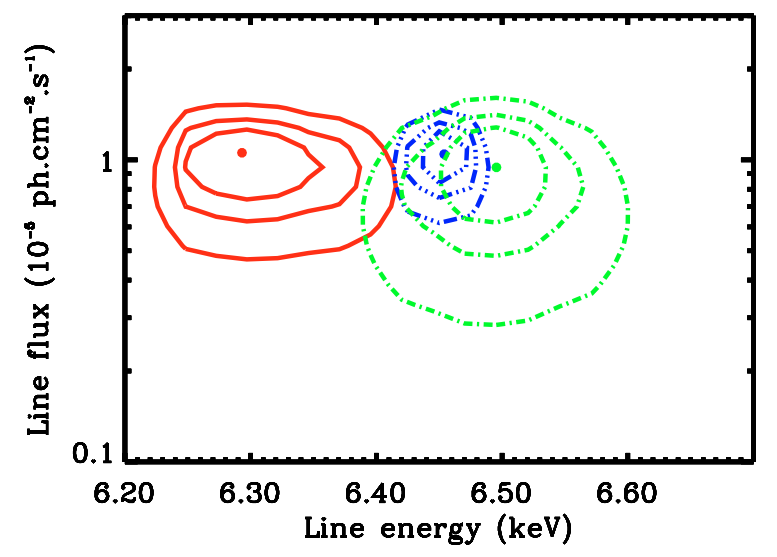

Fig. 9. Contour plots (68, 90 and 99\%) line flux vs. line energy (source frame) of the narrow $(\sigma=0 \mathrm{eV})$ line component added to mimic emission from remote material. OBS 1/2/3: red/solid contour, OBS 4 part 1/2/3: blue/dot-dot-dot-dashed contours, OBS 5: green/dot-dashed contours.

presence of a second narrow line component fixing the parameters of the first line to their best fit values. The best fit parameters for this second narrow line are $E_{\text {line }}=6.48_{-0.06}^{+0.12} \mathrm{keV}$ and $F_{\text {line }}=$ $5.7_{-3.4}^{+2.2} \times 10^{-6} \mathrm{ph} \mathrm{cm}^{-2} \mathrm{~s}^{-1}$. These parameters are now in better agreement with those obtained for OBS 4 and OBS 5.

\subsubsection{Relativistic effects?}

If we believe in the constancy of the narrow line flux, the variability shown by the map excess (Fig. 7) may result from variations in some other underlying component. The most common explanation of such variability is the presence of a broad and rapidly variable line component. This is also illustrated in the upper panel of Fig. 10 where we have plotted the ratio of the complete OBS 4 data spectrum and the best fit power law + DISKLINE + narrow $(\sigma=0 \mathrm{eV})$ Gaussian line model. The DISKLINE model (Fabian et al. 1989) is a relativistic accretion 
Table 4. Best fits parameter values obtained with a power law + relativistic line (DISKLINE) + narrow $(\sigma=0 \mathrm{eV}) \mathrm{Gaussian}$ line model, fitting the data above $3 \mathrm{keV}$. OBS 1,2 and 3 are fitted simultaneously keeping the power law index $\Gamma$, the disc emissivity power law index $q$ and the inner disc radius $r_{\text {in }}$ constant between the different observations. We apply the same procedure for part 1,2 and 3 of OBS 4 . The line energy is given in the source frame, $r_{\text {in }}$ is in unit of $r_{q}$ and is larger than 6 (Schwarzschild metric). The inclination angle is fixed to 30 deg and the outer radius to $1000 r_{g}$.

\begin{tabular}{|c|c|c|c|c|c|c|c|c|c|}
\hline Obs & $\Gamma$ & $q$ & $r_{\text {in }}$ & & $E_{\mathrm{FeK}_{\alpha}}$ & $E W$ & & & $\chi^{2} /$ d.o.f. \\
\hline \multirow[b]{2}{*}{$1 / 2 / 3$} & \multirow[b]{2}{*}{$1.85_{-0.04}^{+0.04}$} & \multirow[b]{2}{*}{$>2.5$} & \multirow[b]{2}{*}{$270_{-70}^{+160}$} & OBS 1 & \multicolumn{2}{|c|}{ OBS 2} & \multicolumn{2}{|c|}{ OBS 3} & \\
\hline & & & & $6.41_{-0.10}^{+0.11} \quad 100_{-50}^{+60}$ & $6.50_{-0.06}^{+0.06}$ & $130_{-40}^{+60}$ & $7.06_{-0.10}^{+0.11}$ & $70_{-40}^{+50}$ & $392 / 424$ \\
\hline & & & & part 1 & \multicolumn{2}{|c|}{ part 2} & \multicolumn{2}{|c|}{ part 3} & \\
\hline 4 part $1 / 2 / 3$ & $1.40_{-0.04}^{+0.04}$ & $>3.9$ & $11_{-2}^{+2}$ & $6.35_{-0.20}^{+0.16} \quad 180_{-110}^{+90}$ & $6.21_{-0.16}^{+0.10}$ & $220_{-90}^{+90}$ & $6.44_{-0.11}^{+0.08}$ & $310_{-110}^{+110}$ & $424 / 442$ \\
\hline 5 & $1.65_{-0.06}^{+0.06}$ & $>5.0$ & $<20$ & & $6.92_{-051}^{+0.37}$ & $230_{-140}^{+140}$ & & & $167 / 158$ \\
\hline
\end{tabular}

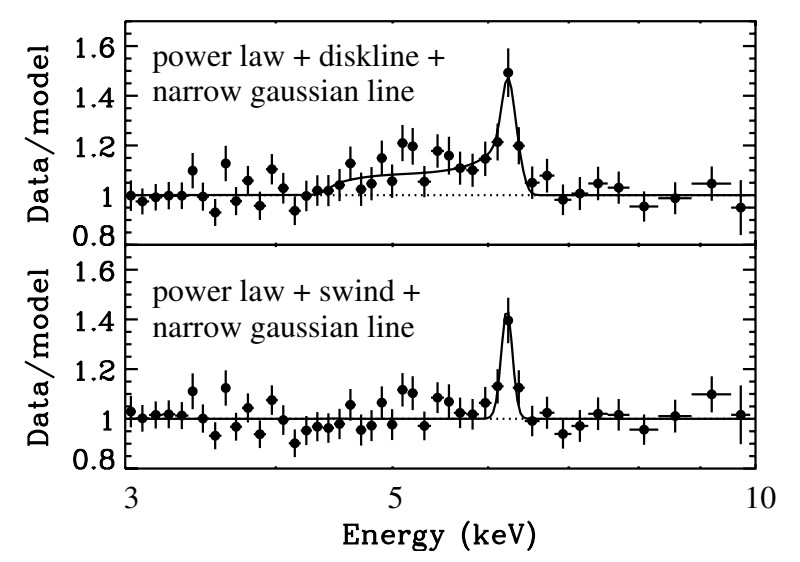

Fig. 10. Ratio data/model for OBS 4. Top: the model is a simple power law + DISKLINE + narrow $(\sigma=0 \mathrm{eV})$ Gaussian line fitted between 3 and $10 \mathrm{keV}$. We ignore the best fit line components (overplotted in solid line) to produce this ratio. Bottom: the model is a simple power law + a partially ionized absorbing material with large velocity shear + narrow $(\sigma=0 \mathrm{eV})$ Gaussian line. We also ignore the best fit line component (over-plotted in solid line) to produce this ratio. The presence of a broad line component is strongly reduced and only the narrow component is present (see Sect. 4.2).

disc line model around a static (Schwarzschild) black hole whose parameters are the line energy, the inner and outer disc radii, the power law index of the disc emissivity law and the disc inclination.

This ratio has been obtained by fitting the data above $3 \mathrm{keV}$ and fixing the inclination to 30 degrees. This figure is then obtained after setting the line normalizations to zero. The presence of a complex line profile is clearly visible with a broad component down to $\sim 4.5 \mathrm{keV}$. The solid line over-plotted in this figure corresponds to the DISKLINE + narrow line shape.

The corresponding best fit values are a power law photon in$\operatorname{dex} \Gamma=1.52_{-0.05}^{+0.06}$, a DISKLINE energy $E_{\mathrm{FeK}_{\alpha}}=6.12_{-0.57}^{+0.46} \mathrm{keV}$, a line $E W$ of $280_{-110}^{+130} \mathrm{eV}$, an inner disc radius $r_{\text {in }}=10.2_{-4.1}^{+13.9} r_{g}$ and a disc emissivity power law index $q>2.4$. The accretion disc outer radius is fixed to $1000 r_{g}$. The fit is very good with a $\chi^{2} /$ d.o.f. $=147 / 165$, suggesting that relativistic effects could indeed be a good explanation of the line profile. We apply this model to the different observations of Mrk 841. During the simultaneous fit of OBS 1, OBS 2 and OBS 3 not only $\Gamma$ but also $q$ ad $r_{\text {in }}$ are kept constant between the different observations. We let the power law normalization as well as the DISKLINE line flux and energy free to vary. We apply the same procedure for the three parts of OBS 4 . Concerning OBS 5, it is analyzed normally as a single observation. We fix the inclination angle to
$30 \mathrm{deg}$ and the outer radius to $1000 r_{g}$. We obtain good fits in all cases. The corresponding best fit parameter values are reported in Table 4.

An alternative to the broad line component could be the presence of ionized warm absorption, smeared by relativistic effects, whose imprints on the underlying continuum could mimic a broad line component (e.g. Reeves et al. 2004). Such a model has also been suggested recently in the literature to produce the soft excess in AGNs and will be discussed more precisely in the next section, but we test this model here to see its impact on the iron line profile. We fit the $3-10 \mathrm{keV}$ data with a simple power law and a partially ionized absorbing material with large velocity shear (SWIND model originally proposed by Gierliński \& Done 2004; and updated by Gierliński \& Done 2006). We also add a narrow Gaussian line to mimic the remote reflection. For comparison with the DISKLINE model, we have plotted in the lower panel of Fig. 10 the ratio data/model for the complete OBS 4 data spectrum. The presence of a broad line component is strongly reduced and only a narrow component is observed. The corresponding best fit values are a power law photon index $\Gamma=1.9_{-0.3}^{+0.5}$, a column density and ionization parameter of the absorbing material $N_{\mathrm{h}}>36 \times 10^{22} \mathrm{~cm}^{-2}$ and $\xi=3080_{-2100}^{+1220}$ and a Gaussian velocity smearing $\sigma=0.19_{-0.05}^{+0.08}$. The fit is statistically acceptable with a $\chi^{2} /$ d.o.f. $=176 / 168$. While it is larger than the DISKLINE fit, both are statistically equivalent following the F-test. We apply this model to the different XMM-Newton observations. We obtain good fits in all cases. The corresponding best fit parameter values are reported in Table 5 .

In conclusion, the data are consistent with the presence of a constant narrow line, potential signature of a remote reflection. Then the apparent line variability shown by the excess map (Fig. 7) may result more likely from slight changes of the underlying continuum. We have tested two possible origins of such variability. It could be due to the presence of a rapidly variable broad iron line component. It could also result from variable relativistically smeared absorption features. Both cases require strong relativistic effects to agree with the data.

\subsection{A broad band physical analysis}

Up to now, our spectral analysis was relatively phenomenological, using simple components to fit the different spectral features present in the data (soft excess, broad and narrow line) and focusing on the high ( $>3 \mathrm{keV}$ ) energy range. The next step is the use of more physical and consistent models on the total energy range of the EPIC-pn instrument.

Recent studies suggest that relativistically blurred (photoionized) reflection from the accretion disc could be an appealing 
Table 5. Best fit parameter values obtained with a power law + relativistic smeared absorption (SWIND) + narrow $(\sigma=0 \mathrm{eV}) \mathrm{Gaussian}$ line model, fitting the data above $3 \mathrm{keV}$. OBS 1,2 and 3 are fitted simultaneously keeping the power law index $\Gamma$ constant between the different observations. We apply the same procedure for part 1,2 and 3 of OBS 4 . The absorbing column density $N_{\mathrm{h}}$ is in unit of $10^{22} \mathrm{~cm}^{-2}$.

\begin{tabular}{|c|c|c|c|c|c|c|c|c|c|c|c|}
\hline Obs & $\Gamma$ & & & & $N_{\mathrm{h}}$ & $\log \xi$ & $\sigma$ & & & & $\chi^{2} /$ d.o.f. \\
\hline $1 / 2 / 3$ & $2.14_{-0.04}^{+0.20}$ & $35.0_{-20.2}^{+4.9}$ & $\begin{array}{c}\text { OBS } 1 \\
3.4_{-0.1}^{+0.1}\end{array}$ & $>0.3$ & $43.4_{-7.5}^{+4.8}$ & $\begin{array}{r}\text { OBS 2 } \\
3.5_{-0.0}^{+0.1}\end{array}$ & $>0.3$ & $>43.6$ & $\begin{array}{l}\text { OBS 3 } \\
3.4_{-0.1}^{+0.1}\end{array}$ & $>0.3$ & $423 / 423$ \\
\hline 4 part $1 / 2 / 3$ & $1.75_{-0.15}^{+0.14}$ & $25.9_{-}^{+}$ & $\begin{array}{c}\text { part 1 } \\
3.3_{-1.0}^{+0.3}\end{array}$ & $0.2_{-0.1}^{+0.2}$ & $8.6_{-1.7}^{+5.8}$ & $\begin{array}{l}\text { part 2 } \\
2.4_{-0.2}^{+0.3}\end{array}$ & \pm & $>29.5$ & $\begin{array}{l}\text { part 3 } \\
3.4_{-0.7}^{+0.1}\end{array}$ & $0.3_{-0.1}^{+0.2}$ & $423 / 441$ \\
\hline 5 & $1.76_{-0.06}^{+0.13}$ & & $<8.0$ & & & $<2.5$ & & & 0 & & $171 / 159$ \\
\hline
\end{tabular}

explanation for the presence of strong soft excesses in AGNs (e.g. Crummy et al. 2006). Moreover, in the cases in which a broad Fe line is clearly detected (such as in MCG-6-30-15) the model is very robust because the soft excess and broad Fe line are fitted self-consistently with the same relativistically blurred reflection model. Crummy et al. (2006) already applied this model to OBS 3 with success. As said in the previous section, an alternative explanation for the origin of the soft excess assumes the presence of absorption features. Since the soft excess does not show strong edges nor absorption lines, strong velocity gradients are needed in the absorbing medium to smear out these features (Gierliński \& Done 2004; Sobolewska \& Done 2007; Schurch \& Done 2006; Gierliński \& Done 2006). A disc wind could produce such spectral signatures. However, this model does not directly explain the presence of a broad line feature and, if present, it has to be produced by another component. On the other hand, as shown in the previous section, ionized absorption may have some impacts on the continuum spectral shape near $6 \mathrm{keV}$ and consequently on the observed line broadness (cf. Fig. 10).

Our XMM-Newton data of Mrk 841 appear to be well adapted to test both interpretations.

\subsubsection{The fitting procedure}

Our base line model has the following components: 1) a neutral absorption free to vary above the galactic value, 2) a cutoff power law continuum (the high energy cut-off being fixed to $300 \mathrm{keV}$ ) and 3) a neutral reflection, if needed, to reproduce the narrow line component. For the neutral reflection we use the tables of the Ross \& Fabian code (Ross \& Fabian 2005) and we fix the ionization parameter to 1 to account for a neutral medium and the illuminating power law continuum to 1.9. This value corresponds to the average $2-10 \mathrm{keV} \mathrm{X-ray} \mathrm{photon} \mathrm{index} \mathrm{of} \mathrm{Seyfert}$ galaxies (e.g. Matt 2001), but the fit results do not depend significantly on this parameter. The free parameters of the first three components of our model are the hydrogen column density, the cut-off continuum power law photon index and normalization and the normalization of the neutral reflection component.

Then we add a fourth component to reproduce the soft excess i.e. either a relativistically blurred ionized reflection or a relativistically smeared ionized absorption (noted REF and ABS respectively in the following). For the ionized reflection, we also use the tables of the Ross \& Fabian code (Ross \& Fabian 2005). The blurring is done by convolving the reflection spectrum with a Laor profile (KDBLUR kernel). The Laor model is a relativistic accretion disc line model around a maximally rotating (Kerr) black hole (Laor 1991). The corresponding free parameters of REF are the inner radius $r_{\text {in }}$ of the reflecting accretion disc, the disc emissivity power law index $q$, the normalization and the ionization parameter $\xi_{\mathrm{REF}}$ of the ionized reflection component. The iron abundance is fixed to the solar one for the computation of the reflection, the disc outer radius to 1000 Schwarzschild radii and the inclination angle to 30 degrees.

Concerning ABS we use again the SWIND model developed by Gierliński \& Done (2006) for XSPEC. The free parameters are the column density $N_{\mathrm{h}}^{\mathrm{ABS}}$ and ionization parameter $\xi_{\mathrm{ABS}}$ of the absorbing wind and the Gaussian velocity smearing $\sigma_{\text {ABS }}$.

We apply these different models to the $0.5-10 \mathrm{keV}$ energy range of the EPIC-pn detectors. Like in the previous section, we analyzed OBS 1, 2, 3 simultaneously. We keep all the parameters constant between the different data sets except the normalization of the cut-off power law continuum and, for REF the normalization and ionization parameters of the ionized reflection, and, for ABS, the column density and ionization parameter of the absorbing wind as well as the Gaussian velocity smearing. We apply the same procedure for the three parts of OBS 4 . Then we have 14 free parameters for REF and 15 for ABS. OBS 5 is analyzed as a single observation with 8 and 7 free parameters for REF and ABS respectively.

\subsubsection{The best fit results}

While these models reproduce relatively well the global spectral shape of the data, the fits are not very good. The residuals show some narrow features especially in the soft band and suggest the presence of absorption. These features are even more pronounced in 2005. Such absorbing material is indeed confirmed by a quick look to the RGS data with the presence of absorption features due to the Unresolved Transition Array of Fe VIIXII. There is also apparently some variability between OBS 4 and OBS 5 indicating some variations of the absorber properties. A detailed analysis of this component is out of the scope of the present paper and will be done in a forthcoming publication (Longinotti et al., in preparation). For the present analysis, we model these absorption features by adding a warm absorber (WA) component (ABSORI in XSPEC) in our fits letting the absorber hydrogen column $N_{\mathrm{h}}^{\mathrm{WA}}$ and ionization state $\xi^{\mathrm{WA}}$ free to vary. The improvement is highly significant. For REF the $\Delta \chi^{2}$ is 30,72 and 75 for the addition of two parameters for OBS $1 / 2 / 3$, OBS 4 part $1 / 2 / 3$ and OBS 5 respectively. For ABS the $\Delta \chi^{2}$ is equal to 15,106 and 70 .

The best fits for both models become now statistically acceptable. The corresponding best fit parameter values for the power law continuum, the neutral reflection and the WA are reported in Tables 6 and 7 for REF and ABS respectively. The best fit parameter values characterizing the ionized reflecting or absorbing material are reported in Tables 8 and 9.

Interestingly, not only the best fits with REF and ABS are statistically acceptable, they are also statistically similar and cannot be ruled out with the present XMM-Newton data. However they predict different spectral characteristics. For instance, 
Table 6. Best fit parameter values for the continuum, the neutral reflection and the WA obtained with the relativistically blurred ionized reflection REF model. $N_{\mathrm{h}}$ is the neutral hydrogen column density, $\Gamma$ the continuum photon index, $N_{\text {neutral }}$ the neutral reflection normalization, $N_{\mathrm{h}}^{\mathrm{WA}}$ the ionized hydrogen column density of the WA and $\xi^{\mathrm{WA}}$ its ionization parameter. $\Delta \chi^{2}$ is the fit improvement with the addition of the warm absorber. The corresponding best fit parameter values of the reflecting material are reported in Table 8 .

\begin{tabular}{lccccccc}
\hline \hline Obs. & $\begin{array}{c}N_{\mathrm{h}} \\
10^{20} \mathrm{~cm}^{-2}\end{array}$ & $\Gamma$ & $\begin{array}{c}N_{\text {neutral }} \\
\times 10^{5}\end{array}$ & $\begin{array}{c}N_{\mathrm{h}}^{\mathrm{WA}} \\
10^{22} \mathrm{~cm}^{-2}\end{array}$ & $\xi^{\mathrm{WA}}$ & $\Delta \chi^{2}$ & $\chi^{2} /$ d.o.f. \\
\hline $1 / 2 / 3$ & $<2.8$ & $2.45_{-0.04}^{+0.05}$ & $7.9_{-1.5}^{+2.0}$ & $1.1_{-0.4}^{+0.1}$ & $>900$ & 30 & $783 / 788$ \\
$4-1 / 2 / 3$ & $7.4_{-0.9}^{+1.1}$ & $1.59_{-0.02}^{+0.01}$ & $6.4_{-1.5}^{+1.4}$ & $2.7_{+0.8}^{-0.5}$ & $1500_{+470}^{-350}$ & 72 & $865 / 809$ \\
5 & $6.5_{-1.6}^{+4.0}$ & $2.01_{-0.09}^{+0.05}$ & $5.8_{-2.3}^{+2.4}$ & $0.4_{-0.4}^{+0.3}$ & $50_{-10}^{+10}$ & 75 & $303 / 278$ \\
\hline
\end{tabular}

Table 7. Best fit parameter values for the continuum, the neutral reflection and the WA obtained with the relativistically smeared ionized absorption ABS model. The parameters definition is the same as in Table 6. The corresponding best fit parameter values of the absorbing material are reported in Table 9.

\begin{tabular}{lccccccc}
\hline \hline Obs. & $\begin{array}{c}N_{\mathrm{h}} \\
10^{20} \mathrm{~cm}^{-2}\end{array}$ & $\Gamma$ & $\begin{array}{c}N_{\text {neutral }} \\
\times 10^{5}\end{array}$ & $\begin{array}{c}N_{\mathrm{h}}^{\mathrm{WA}} \\
10^{22} \mathrm{~cm}^{-2}\end{array}$ & $\xi^{\mathrm{WA}}$ & $\Delta \chi^{2}$ & $\chi^{2} /$ d.o.f. \\
\hline $1 / 2 / 3$ & $<2.6$ & $2.22_{-0.01}^{+0.03}$ & $\begin{array}{c}10.7_{-2.0}^{+2.0} \\
0.10_{-0.03}^{+0.01}\end{array}$ & $60_{-20}^{+30}$ & 15 & $824 / 787$ \\
$4-1 / 2 / 3$ & $<2.8$ & $1.90_{-0.04}^{+0.04}$ & $7.7_{-1.5}^{+1.6}$ & $0.2_{-0.1}^{+0.1}$ & $20_{-10}^{+15}$ & 106 & $843 / 808$ \\
5 & $<6.0$ & $1.96_{-0.02}^{+0.02}$ & $7.0_{-2.5}^{+2.5}$ & $0.4_{-0.1}^{+0.1}$ & $40_{-10}^{+20}$ & 70 & $315 / 279$ \\
\hline
\end{tabular}

Table 8. Best fit parameter values characterizing the ionized reflecting material in the REF model. $\xi_{\text {REF }}$ is the ionization parameter of the ionized reflection component, $q$ the disc emissivity power law index and $r_{\text {in }}$ the inner radius of the reflecting accretion disc. The reflection fraction is the ratio of the ionized reflection flux in the $0.1-1000 \mathrm{keV}$ band divided by the total $0.1-1000 \mathrm{keV}$ flux of the best fit model.

\begin{tabular}{|c|c|c|c|c|c|c|}
\hline Obs. & & $\xi_{\text {REF }}$ & & $q$ & $\begin{array}{l}r_{\text {in }} \\
r_{g} \\
\end{array}$ & $\overline{\text { Refl. frac. }}$ \\
\hline $1 / 2 / 3$ & $\begin{array}{l}\text { OBS 1 } \\
130_{-10}^{+10}\end{array}$ & $\begin{array}{l}\text { OBS 2 } \\
155_{-10}^{+20}\end{array}$ & $\begin{array}{l}\text { OBS 3 } \\
100_{-10}^{+10}\end{array}$ & $>8.6$ & $<1.4$ & $30 \%$ \\
\hline $4-1 / 2 / 3$ & $\begin{array}{c}\text { part 1 } \\
315_{-10}^{+10}\end{array}$ & $\begin{array}{l}\text { part 2 } \\
325_{-10}^{+10}\end{array}$ & $\begin{array}{c}\text { part 3 } \\
200_{-40}^{+40}\end{array}$ & $3.7_{-0.2}^{+0.2}$ & $<2.5$ & $38 \%$ \\
\hline 5 & & $80_{-20}^{+40}$ & & $4.3_{-0.4}^{+0.4}$ & $<2.7$ & $43 \%$ \\
\hline
\end{tabular}

the continuum spectral variability between 2001 and 2005 is significantly larger with REF $(\Delta \Gamma=0.9)$ compared to ABS $(\Delta \Gamma=$ 0.3 ). The former case is relatively unusual for a Seyfert 1 galaxy and would require strong changes in the emitting regions. The properties of the WA are also different, at least for OBS $1 / 2 / 3$ and OBS 4 , the column density and ionization parameters being larger by a factor 10 (and even more for $\xi^{\mathrm{WA}}$ ) between REF and ABS. On the other hand, the neutral reflection normalization is consistent with a constant between the different pointings with both models, supporting the presence of remote reflection.

For comparison we have plotted the unfolded best fits obtained with REF and ABS in Fig. 11 for OBS 1, the first part of OBS 4 and OBS 5. We have also over-plotted the different spectral components like the neutral reflection and, for the REF model, the relativistically blurred ionized reflection. Large differences above $10 \mathrm{keV}$ are expected especially for 2001 and Jan. 2005.

We can also analyze more precisely the results obtained for each model separately, beginning with REF. Interestingly, while this model requires still extreme values of the disc inner radius, the disc emissivity power law index, now also constrained by the soft excess, is close to 4 in OBS 4 part $1 / 2 / 3$ and OBS 5, in better agreement with theoretical expectations (e.g. Martocchia et al. 2000). This contrasts with the results obtained with the DISKLINE model where larger values of $q$ are generally found (see Table 4). We agree that these discrepancies can be partly explained by intrinsic differences between the LAOR and DISKLINE profiles. Note however that $q$ is still very large $(>8.6)$ in OBS $1 / 2 / 3$ whatever the relativistic line profile used. The photon index is also very steep $(\Gamma \sim 2.45)$ in these observations. Noticeably, constraining $q$ to be smaller than 5 gives a more reasonable photon index with $\Gamma=2.26_{-0.05}^{+0.07}$ while the other parameters do not significantly change. The $\chi^{2}$ is larger $\left(\chi^{2} /\right.$ d.o.f. $=804 / 787$ i.e. $\Delta \chi^{2}=$ 20) but still acceptable.

We have also reported in Table 8 the reflection fraction, i.e. the ratio between the flux in the ionized reflection component and the total flux. It is of the order of $30-50 \%$ which is relatively large since we expect $\sim 10-20 \%$ in the case of an isotropic illumination. Finally the properties of the WA significantly change for OBS 5 compared to the other observations.

On the other hand, the characteristics of the WA are relatively constant between the different pointings with the ABS model. The continuum power law index is also close to the standard one for a Seyfert galaxy i.e. $~ 1.9$, reaching also a steeper value $(\sim 2.2)$ in 2001 . To fit the data the ABS model requires a change in the properties of the relativistically smeared absorbing material between 2001 and 2005, the column density and the velocity smearing being larger in 2001 . These parameters are more consistent with each other between OBS 4 and OBS 5.

\subsubsection{Comparison with a simultaneous BeppoSAX observation}

The BeppoSAX instruments cover the $0.1-200 \mathrm{keV}$ range and thus better constrains the reflection component compared to XMM-Newton. Mrk 841 was pointed by BeppoSAX between the 11th and 14th of January 2001, i.e. partly simultaneously with the XMM-Newton observations of 2001, for a total net time exposure of $\sim 90 \mathrm{ks}$ for the MECS instrument $(2-10 \mathrm{keV}$ energy range) $\sim 40 \mathrm{ks}$ for the PDS (10-200 keV energy range) and only $20 \mathrm{ks}$ for the LECS (0.1-2 keV energy range). Since the BeppoSAX observation was much longer than the XMM-Newton one (see Fig. 1 of Petrucci et al. 2002), we did not try to fit the XMM-Newton and BeppoSAX data altogether. 
Table 9. Best fit parameter values characterizing the absorbing material in the ABS model. $N_{\mathrm{h}}^{\mathrm{ABS}}$ and $\xi_{\mathrm{ABS}}$ are the column density and ionization parameter of the absorbing wind and $\sigma_{\mathrm{ABS}}$ the Gaussian velocity smearing.

\begin{tabular}{|c|c|c|c|c|c|c|c|c|c|}
\hline Obs. & & $\begin{array}{c}N_{\mathrm{h}}^{\mathrm{ABS}} \\
10^{22} \mathrm{~cm}^{-2}\end{array}$ & & & $\log \left(\xi_{\mathrm{ABS}}\right)$ & & & $\begin{array}{c}\sigma_{\mathrm{ABS}} \\
\mathrm{c}\end{array}$ & \\
\hline & OBS 1 & OBS 2 & OBS 3 & OBS 1 & OBS 2 & OBS 3 & OBS 1 & OBS 2 & OBS 3 \\
\hline $1 / 2 / 3$ & $23.1_{-0.7}^{+2.3}$ & $24.4_{-1.5}^{+1.5}$ & $14.3_{-1.1}^{+1.3}$ & $3.22_{-0.03}^{+0.05}$ & $3.27_{-0.03}^{+0.04}$ & $3.01_{-0.03}^{+0.02}$ & $>0.4$ & $0.36_{-0.04}^{+0.05}$ & $>0.45$ \\
\hline $4-1 / 2 / 3$ & $\begin{array}{c}\text { part 1 } \\
15.5_{-1.3}^{+2.0}\end{array}$ & $\begin{array}{c}\text { part 2 } \\
20.4_{-1.3}^{+3.3}\end{array}$ & $\begin{array}{c}\text { part 3 } \\
17.5_{-2.0}^{+1.5}\end{array}$ & $\begin{array}{c}\text { part 1 } \\
2.90_{-0.05}^{+0.04}\end{array}$ & $\begin{array}{c}\text { part 2 } \\
2.99_{-0.05}^{+0.06}\end{array}$ & $\begin{array}{c}\text { part } 3 \\
2.90_{-0.05}^{+0.05}\end{array}$ & $\begin{array}{c}\text { part 1 } \\
0.25_{-0.03}^{+0.03}\end{array}$ & $\begin{array}{c}\text { part 2 } \\
0.29_{-0.03}^{+0.04}\end{array}$ & $\begin{array}{c}\text { part } 3 \\
0.26_{-0.03}^{+0.04}\end{array}$ \\
\hline 5 & & $16.7_{-8.6}^{+5.9}$ & & & $3.22_{-0.18}^{+0.16}$ & & & $0.30_{-0.04}^{+0.10}$ & \\
\hline
\end{tabular}

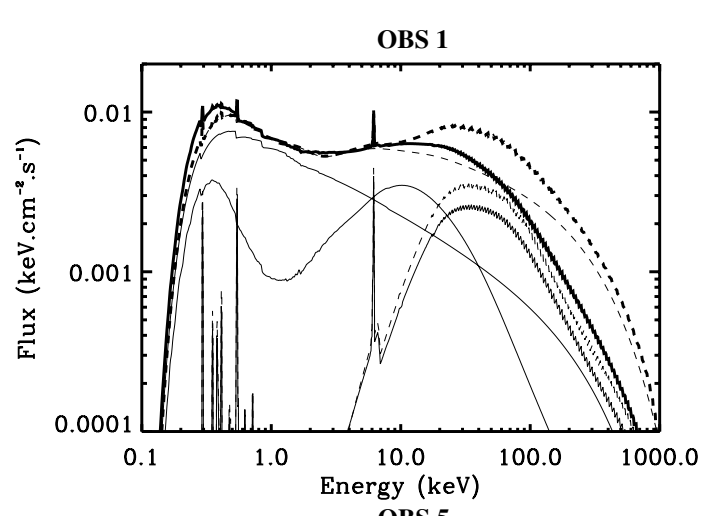

OBS 5

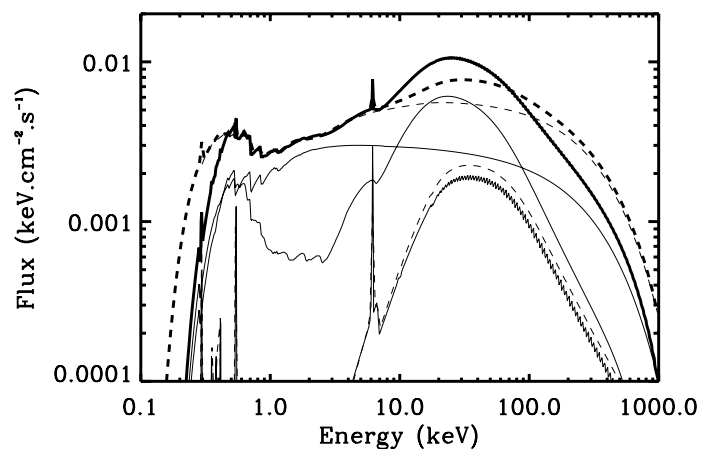

We only checked the consistency of the BeppoSAX data above $10 \mathrm{keV}$ with the XMM-Newton best fit model expectation.

The unfolded best fit spectra obtained with REF and ABS for OBS 1 are plotted in Fig. 12 with the BeppoSAX MECS and PDS data (we ignore the LECS data due to their poor quality). Only the MECS normalization was let free to vary, the PDS one being fixed to 0.86 the MECS one (following Fiore et al. 1999). The agreement is relatively good given the possible variability of the reflection component during the BeppoSAX pointing and the fixed value of $300 \mathrm{keV}$ of the power law continuum high energy cut-off in the fits of the XMM-Newton data. We note however a better agreement of the ABS model with the BeppoSAX /PDS data above $10 \mathrm{keV}$. With the REF model, the flux above $10 \mathrm{keV}$ is underestimated by a factor $\sim 2$. However this depends on some of our model assumptions. For example constraining $q$ to be smaller than 5 instead of being completely free (see Sect. 4.3.2) gives a better agreement (the green data points on the left plot) with the BeppoSAX/PDS data, similar to the agreement found with ABS.

\section{Indication of redshifted narrow iron lines}

\subsection{A strongly redshifted line at $4.8 \mathrm{keV}$ in OBS 4}

We focus here on a possible detection of a narrow line at $4.8 \mathrm{keV}$ in the first part of OBS 4. This feature is clearly visible in Fig. 5

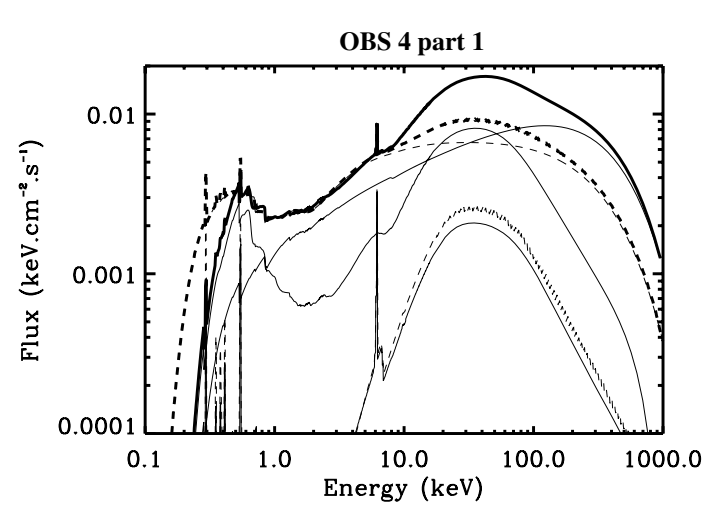

Fig. 11. Comparison of the unfolded best fits obtained with the REF and ABS models for OBS 1, the first part of OBS 4 and OBS 5. The thick solid lines correspond to the total best fit REF model and the dashed thick line to the ABS one. Overplotted in these figures in thin lines are the different components of each model. Thin solid lines: cut-off power law continuum, relativistically blurred ionized reflection and neutral reflection. Thin dashed lines: cut-off power law continuum, modified by relativistically smeared ionized absorption, and neutral reflection. The fluxes are in $\mathrm{keV} \mathrm{cm}^{-2} \mathrm{~s}^{-1}=1.6 \times 10^{-9} \mathrm{erg} \mathrm{cm}^{-2} \mathrm{~s}^{-1}$.

but also on the excess map shown in Fig. 7. To estimate its confidence level we first added a Gaussian with energy $\sim 4.8 \mathrm{keV}$ to a simple power law best fit model of OBS 4 part 1 . The Gaussian energy and flux are let free to vary but, given the apparent narrowness of the feature, we fix the Gaussian width to zero. The excess is clearly detected with an improvement of the fit of $\Delta \chi^{2}=11$. The best fit parameters of the line are $E_{\text {line }}=4.80_{-0.03}^{+0.03} \mathrm{keV}$ (source frame) and an equivalent width $E W=50 \pm 20 \mathrm{eV}$. The contour plot of the line energy versus the line flux is plotted in solid line in Fig. 13.

We also estimate the confidence level of this detection by simulating a large number of spectra with a continuum similar to that of OBS 4 part 1 . We fit the different simulations with a simple power law. We then add a Gaussian, fixing its energy between 4 and $8 \mathrm{keV}$ by step of $0.1 \mathrm{keV}$ and fit again the data, the Gaussian normalization being free to vary while the Gaussian width was fixed to 0 . Then for each simulated spectra, we keep the best $\Delta \chi^{2}$ resulting from this procedure. For 1000 simulations we only find 14 cases where a $\Delta \chi^{2}>11$ corresponding to a confidence level of $98.5 \%$. The strong detection in the EPIC-pn data is however attenuated by the weak detection in the MOS data. Indeed we have plotted in dashed line in Fig. 6 the corresponding contour plot when fitting the EPIC-pn and MOS data simultaneously. The detection is now significant at only $84.4 \%$ from our simulations. 

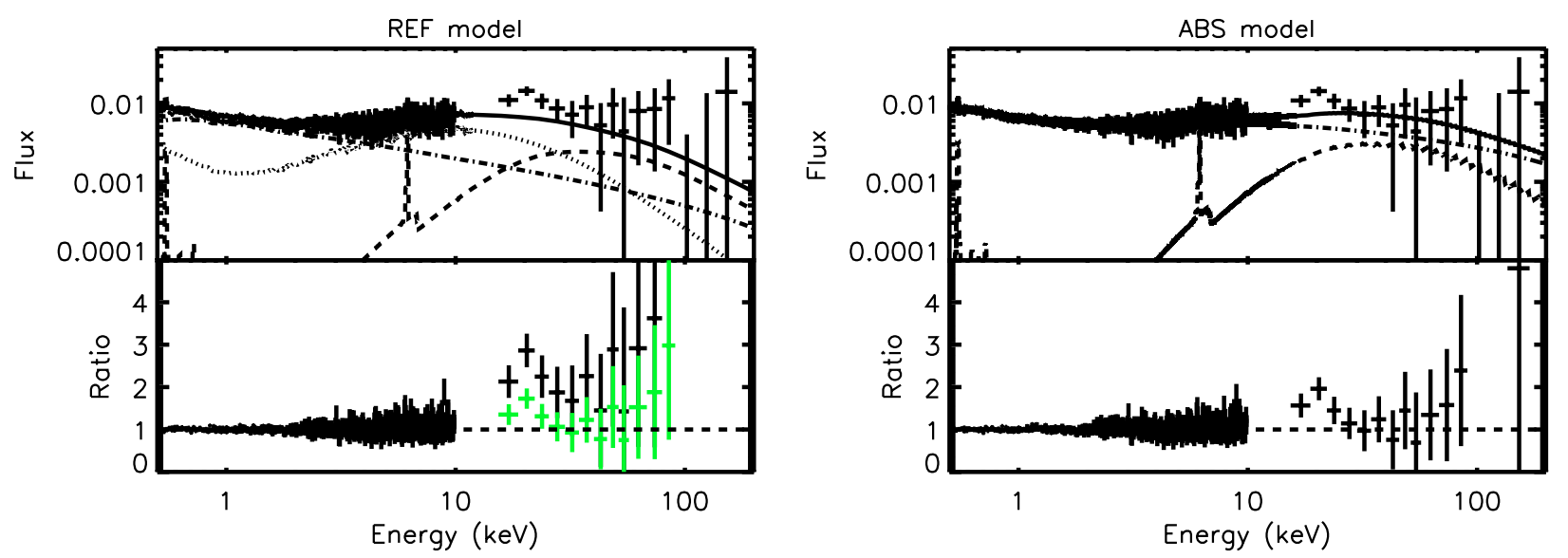

Fig. 12. Comparison of the OBS 1 best fit obtained with the REF (left) and ABS (right) models with the simultaneous BeppoSAX data. The different spectral components are over-plotted in the upper plots: left: neutral reflection (dashed line), ionized blurred reflection (dotted line), cutoff power law (dot-dashed line) and total (solid line). The grey (green on the colored version) ratios correspond to the best fit REF model with the disc emissivity power law index $q$ forced to be smaller than 5 (see Sect. 4.3.2); right: neutral reflection (dashed line), cut-off power law modified by smeared absorption (dot-dashed line) and total (solid line).

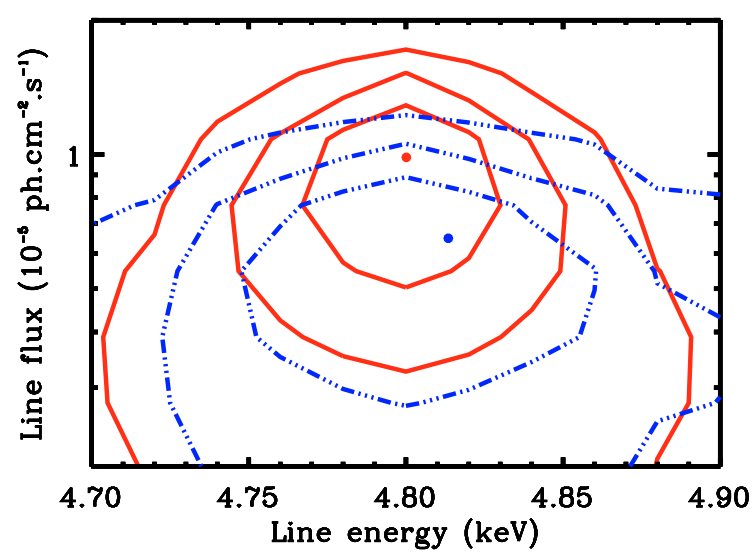

Fig. 13. Contour plot energy (source frame) vs. flux of the line detected close to $4.8 \mathrm{keV}$ during part 1 of OBS 4 . The contours correspond to 68,90 and $99 \%$ The solid and dash-dotted contours correspond to the EPIC-pn and EPIC-pn + EPIC-mos data respectively.

If we assume that this line is a redshifted neutral fluorescent iron line, its observation at $4.8 \mathrm{keV}$ (source frame) implies a redshift factor of $\sim 0.75$. Such high redshift value suggests an origin close to the central engine where relativistic effects become important. Moreover, the narrow shape of the line constrains the emitting region to be sufficiently small. There are growing evidences of such variable narrow emission features in the X-ray spectra of several AGN in the literature (Turner et al. 2004; Porquet et al. 2004; Petrucci et al. 2002; Tombesi et al. 2007). The common scheme to explain these components suppose the presence of transient magnetic flares briefly illuminating a localized part (hot spot) of the accretion disc and producing the iron line by fluorescence.

Following Longinotti et al. (2004, Fig. 4) we have produced a $90 \%$ probability map (from the $90 \%$ error on the line energy) of the accretion disc where the line could come from, for different values of the black hole specific momentum and disc inclination angles (cf. Fig. 14). For an inclination of $25 \mathrm{deg}$ the line emission can come from region at a distance as small as $3 r_{g}$ from the central black hole and even closer for larger inclinations. Moreover, if we assume that we observed the same hot spot on the disc for more than an orbit then the global line profile is expected to be

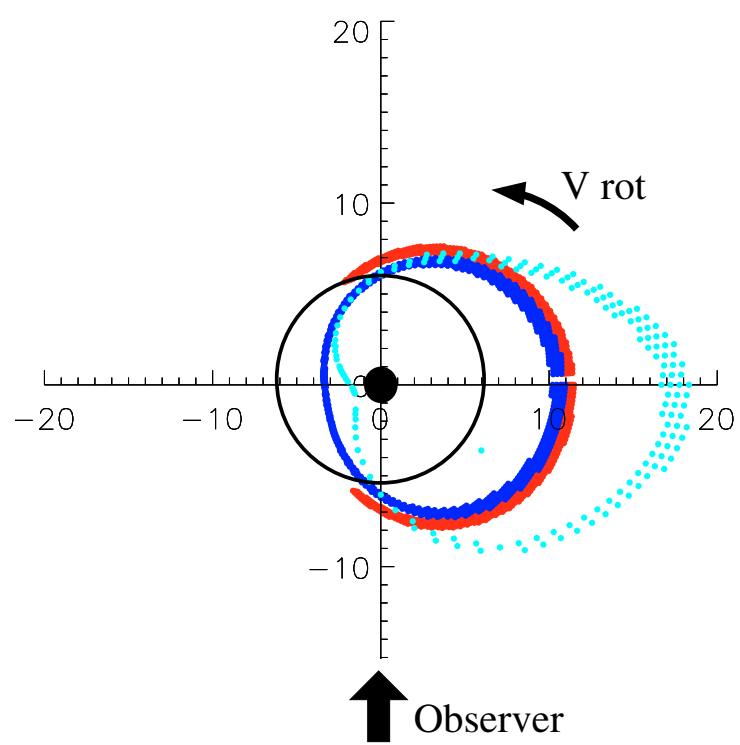

Fig. 14. A schematic map of the inner accretion disc showing the allowed regions where a narrow line with parameters consistent with the one observed during part $1\left(E_{\text {line }}=4.79_{-0.02}^{+0.04} \mathrm{keV}\right)$ could be emitted. In red: $a=0$ and $i=25$. In blue: $a=0.998$ and $i=25$. In cyan: $a=0.998$ and $i=60 \mathrm{deg}$. The black circle correspond to a radius of $6 r_{g}$. The disc is rotating counterclockwise.

relatively complex (e.g. Dovčiak et al. 2004) with a strong blue peak. If we interpret the observed feature with this blue peak, a Kerr solution is unavoidable (Pecháček et al. 2005).

\subsection{A slightly redshifted line at $6.2 \mathrm{keV}$ in OBS 1}

We discuss now the possible detection of a narrow line feature near $6.2 \mathrm{keV}$ (source frame) in the first observation of 2001 as already noted in Sect. 4.2.2. Starting from the best fit DISKLINE model reported in Table 4, we add a narrow $(\sigma=0 \mathrm{eV})$ Gaussian line letting its energy free to vary for each data set OBS 1, OBS 2 and OBS 3. This differs from the analysis done in Sect. 4.2.2 where the narrow Gaussian line parameters were kept constant between the different observations. The corresponding energy vs. flux contour plot of each narrow line are reported in 


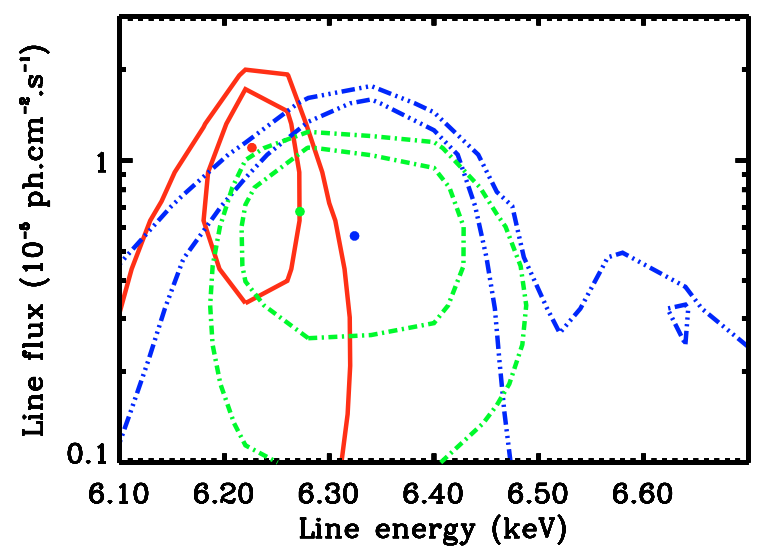

Fig. 15. Contour plot (68 and $90 \%$ ) energy (source frame) vs. flux of the narrow line $(\sigma=0 \mathrm{eV})$ added to the DISKLINE fit of OBS 1 (solid red line), OBS 2 (dotdotdot-dashed blue line) and OBS 3 (dot-dashed green line). The narrow line energy in OBS 1 peaked close to $6.2 \mathrm{keV}$ (source frame).

Fig. 15. The narrow line energy is consistent with the neutral fluorescent iron line energy of $6.4 \mathrm{keV}$ in OBS 2 and 3 but is inconsistent at more than $90 \%$ with this value for OBS 1 where $E_{\text {line }}=6.23_{-0.03}^{+0.05} \mathrm{keV}$.

If interpreted, as above, as a (slightly) redshifted narrow iron line we can also produced a $90 \%$ probability maps of the accretion disc where the line could come from, for different values of the black hole specific momentum and disc inclination angles. This has been done in Fig. 16.

\section{Summary and discussion}

We have presented in this paper a detailed spectral analysis of the XMM-Newton/EPIC-pn data of Mrk 841 including all the XMM-Newton observations of this object. Given the relative spectral complexity of this source we would like to summarize first the different results we obtained.

Mrk 841 has been observed three times in January 2001 (OBS 1, OBS 2 and OBS 3) and two times in 2005 in January (OBS 4) and July (OBS 5). Flux and spectral variations being present during the $\sim 45 \mathrm{ks}$ of OBS 4 , this observation has been divided in 3 parts for the spectral analysis. A strong soft excess as well as a complex iron line profile, both known to be present in this source for a long time, are clearly detected in all these pointings, the high sensitivity of the XMM-Newton/EPIC-pn unveiling very puzzling spectral and temporal behavior. The $0.5-10 \mathrm{keV}$ flux varies by a factor 3 in 4 years and is dominated by the soft band $(<3 \mathrm{keV})$ variability, the data above $\sim 5 \mathrm{keV}$ keeping roughly constant on short and long time scales. The spectral variability is also important. Fitting the 3-10 keV data with a simple power law the spectral index varies from $\sim 1.9$ in 2001 down to $\sim 1.3$ in Jan. 2005. However a simple pivoting power law component cannot explain by itself the broadband $0.5-10 \mathrm{keV}$ spectral variability observed on year time scale thus indicating a more complex spectral variability.

The line profile is also complex, being apparently a mixture of broad and narrow components. It is highly variable and the 2005 data confirm the rapid line variability observed in 2001 by Petrucci et al. (2002) and Longinotti et al. (2004) with a variability time scale as short as a few kilo-seconds. This strong $\mathrm{X}$-ray variability suggests a small emitting region close to the black hole. Fitting the line with a DISKLINE model gives a good

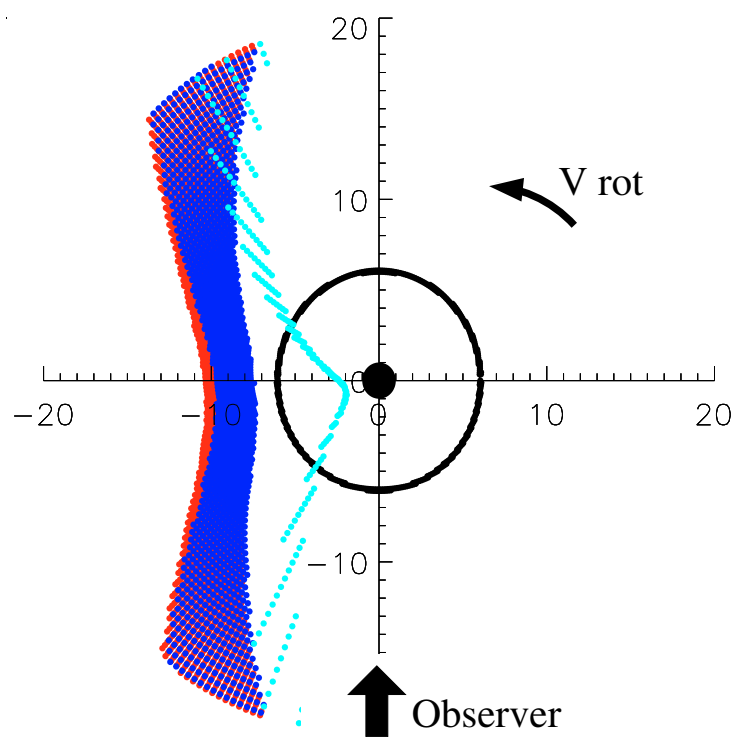

Fig. 16. Same as Fig. 14 but for a narrow line with parameters consistent with the one observed during OBS $1\left(E_{\text {line }}=6.23_{-0.03}^{+0.05} \mathrm{keV}\right)$. We have limited ourselves to the inner part $\left(<20 r_{q}\right)$ of the accretion disc. Cf. Fig. 14 for the color scheme. The blue and red regions overlapped almost totally.

representation of the line profile and requires in some cases steep disc emissivity power law indexes.

Given the high complexity of the spectra, we choose to decrease the degree of freedom of some of our fits by fitting simultaneously observations with roughly the same underlying continuum, e.g. the three pointings of 2001 as well as the different parts of OBS 4 . In these cases, during the fits the power law continuum was kept constant in shape but not in flux between the data sets. Concerning OBS 5, it was analyzed normally as a single observation. A narrow line component is present in all the pointings and its flux is consistent with a constant between 2001 and 2005. This suggests the presence of remote reflection in the data. If this interpretation is correct, then the observed variability of the iron line complex is more likely due to the variability of the underlying component below the narrow iron line, either due to a broad line component or strong smeared absorption.

Then we analyze the broad band $(0.5-10 \mathrm{keV})$ EPIC-pn energy range. We applied two different models, recently proposed in the literature, for the origin of the soft excess: a relativistically blurred ionized reflection (REF) and a relativistically smeared ionized absorption (ABS)). We also added a neutral reflection to reproduce the narrow line component. Both models are a reasonable representation of the overall broad band shape but are formally statistically unacceptable du to the presence of absorption features in the soft energy range. The addition of a warm absorber strongly improves the fits that converge to statistically acceptable and statistically equivalent representations of the data. Both models are also consistent with the partly simultaneous BeppoSAX observations done in 2001.

Finally we also note the presence of a strong narrow feature near $4.8 \mathrm{keV}$ in the first part of OBS 4 . Its detection is marginally significant in the EPIC-pn data $(>98.5 \%)$ but the significance decreases to $84 \%$ when we include the MOS data. If interpreted as the blue horn of a relativistically distorted neutral iron line, the large redshift implies the presence of a Kerr black hole.

The most remarkable result of our study is to reproduce reasonably well the broad band $(0.5-10 \mathrm{keV})$ and complex spectral characteristics of Mrk 841 with a small number of spectral 


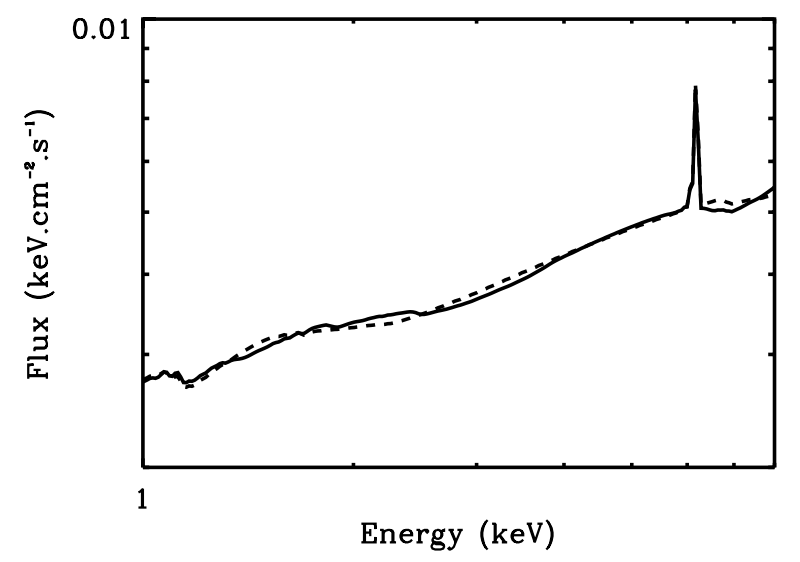

Fig. 17. Unfolded best fits of OBS 5 obtained with REF (solid line) and ABS (dashed line) in the $1-8 \mathrm{keV}$ range. The curvature of the spectral shape below to the iron line, due to a broad line component in REF and by smeared absorption in ABS, is present in both cases.

components. However we were not able to discriminate between the two different interpretations of the soft X-ray excess, which have been debated in the recent literature. Moreover both models are able to reproduce the spectral shape close to the iron line either by adding a broad component, like in the case of the REF model, or by modifying the power law shape, like in the the ABS model. This is exemplified in Fig. 17 where we show a zoom of the unfolded best fit models obtained with REF and ABS for OBS 5 in the $1-8 \mathrm{keV}$ range.

Whatever the model used, the need of strong relativistic effects to smear/blur the absorption/emission features suggest an origin of the X-ray emission close to the central black hole. Then the absorbing material required in the ABS model could be the base of a disc wind/jet surrounding the inner X-ray emitting region. The external part of this wind, with lower velocities, may then explain the absorption features of the WA.

On the other hand, the REF model is based on the presence of strong reflection as indicated by the large reflection fraction that we obtained to fit the data. Another way of quantifying this large amount of reflection is to measure the commonly used reflection parameter $R$. Since this is not a direct parameter of the reflection tables that we used, we estimate its value by roughly adjusting the reflection shape (ignoring the blurring effects) with the one expected with the PEXRIV model of XSPEC fixing the power law photon index and normalization as well as the ionization parameter of PEXRIV to our best fit values. Following this method, we obtain $R$ values of the order of 10,2 and 4 for OBS $1 / 2 / 3$, OBS 4 and OBS 5 respectively. We recall here that the blurring effects, as well as the ionization level of the reflecting material considerably smooth the reflection shape. In consequence even a reflection component with $R=10$ is strongly attenuated in the outgoing spectrum. But such large reflections necessarily require the presence of some effects that increase the reflection component compared to the illuminating continuum like e.g. in the light bending effects model (Miniutti et al. 2003; Miniutti \& Fabian 2004 ) or the inhomogeneous accretion flows model proposed recently by Merloni et al. (2006).

The low statistics of our rms spectra (cf. Fig. 2) do not allow a detailed comparison with the variability behavior expected with ABS or REF and thus unable us to discriminate the two models. We can note however that, while both models can correctly reproduce the rms spectra of some AGNs (e.g. Gierliński \& Done 2006; Ponti et al. 2006), ABS predicts an rms spectrum which rapidly increases and peaks around $1 \mathrm{keV}$, where the absorber enhances the variability (Gierliński \& Done 2006). This is not seen in our rms spectra and so argues against this model a bit or at least that there is no absorber variability on short timescales. But this has to be tested on data of better quality.

Finally, we note that our (admittedly marginal) detection of redshifted narrow iron lines in the data better fits in the relativistically blurred reflection interpretation where such features are naturally expected. But we agree that this is a relatively weak argument that requires further investigations.

\section{Conclusion}

Mrk 841 is a bright Seyfert 1 galaxy which is known to possess a strong soft excess and complex iron line profile. We have presented a detailed analysis of the whole XMM-Newton/EPIC-pn data of this object from 2001 to 2005. The conclusions of this analysis can be summed up as follows:

- Strong flux and spectral variability are observed on month and year time scales. The $0.5-10 \mathrm{keV}$ flux varies by a factor 3 in 4 years and is dominated by the soft band $(<3 \mathrm{keV})$ variability, the data above $\sim 5 \mathrm{keV}$ keeping roughly constant on short and long time scales.

- The iron line is apparently a mixture of broad and narrow components. Its profile is rapidly varying on very short time scale (a few ks).

- The broad band 0.5-10 keV spectrum is well described by a model including 1) a neutral absorption; 2) a cut-off power law continuum; 3 ) a neutral reflection and a fourth component for the soft excess. We use two different models for this last component: a relativistically blurred photoionized reflection (REF model) and a relativistically smeared ionized absorption (ABS model). Both models give statistically acceptable and statistically equivalent fits of the data even including, for 2001 observations, partly simultaneous BeppoSAX data up to $200 \mathrm{keV}$.

- Both models agree with the presence of remote reflection characterized by a constant narrow component in the data. However they differ on the presence of a broad line component present in REF but not needed in ABS. Consequently, the physical interpretation of the line profile variability is quite different, resulting from the variability of the broad line component in REF and from the variability of the absorbing medium in ABS).

- Mrk 841 is also a good candidate for the observation of redshifted narrow iron lines, two marginal detections being discussed in this paper. If such features would favor the relativistically blurred photoionized reflection interpretation, their marginal detections do not permit any clear conclusion.

While it seems reasonable that the reality is a complex combination of absorption and reflection effects the present analysis underlines the difficulty in well disentangling these components in the X-ray spectra of AGN. Broad band observations including data above $10 \mathrm{keV}$ (Suzaku, INTEGRAL) will certainly help to better test both interpretations but the high sensitivity in the $10-50 \mathrm{keV}$ energy range is crucial. We may need to wait for missions like SIMBOL-X or XEUS to have a clear understanding of what processes really take place in sources like Mrk 841.

\section{References}

Arnaud, K. A., Branduardi-Raymont, G., Culhane, J. L., et al. 1985, MNRAS, 217, 105

Bianchi, S., Matt, G., Haardt, F., et al. 2001, A\&A, 376, 77 
Chevallier, L., Collin, S., Dumont, A.-M., et al. 2006, A\&A, 449, 493 Crummy, J., Fabian, A. C., Gallo, L., \& Ross, R. R. 2006, MNRAS, 365, 1067 Czerny, B., Nikołajuk, M., Różańska, A., et al. 2003, A\&A, 412, 317

Day, C. S. R., Fabian, A. C., George, I. M., \& Kunieda, H. 1990, MNRAS, 247, $15 \mathrm{P}$

Dovčiak, M., Bianchi, S., Guainazzi, M., Karas, V., \& Matt, G. 2004, MNRAS, 350,745

Fabian, A. C., Rees, M. J., Stella, L., \& White, N. E. 1989, MNRAS, 238, 729

Fabian, A. C., Miniutti, G., Gallo, L., et al. 2004, MNRAS, 353, 1071

Falco, E. E., Kurtz, M. J., Geller, M. J., et al. 1999, PASP, 111, 438

Fiore, F., Guainazzi, M., \& Grandi, P. 1999, Cookbook for BeppoSAX NFI Spectral Analysis

George, I. M., \& Fabian, A. C. 1991, MNRAS, 249, 352

George, I. M., Nandra, K., Fabian, A. C., et al. 1993, MNRAS, 260, 111

Gierliński, M., \& Done, C. 2004, MNRAS, 349, L7

Gierliński, M., \& Done, C. 2006, MNRAS, 371, L16

Haardt, F., Maraschi, L., \& Ghisellini, G. 1994, ApJ, 432, L95

Iwasawa, K., Miniutti, G., \& Fabian, A. C. 2004, MNRAS, 355, 1073

Jansen, F., Lumb, D., Altieri, B., et al. 2001, A\&A, 365, L1

Johnson, W. N., Zdziarski, A. A., Madejski, G. M., et al. 1997, in Proceedings of the Fourth Compton Symposium, ed. C. D. Dermer, M. S. Strickman, \& J. D. Kurfess, AIP Conf. Proc., 410, 283

Kinney, A. L., Antonucci, R. R. J., Ward, M. J., Wilson, A. S., \& Whittle, M. 1991, ApJ, 377, 100

Laor, A. 1991, ApJ, 376, 90

Larsson, J., Fabian, A. C., Miniutti, G., \& Ross, R. R. 2007, MNRAS, 376, 348

Longinotti, A. L., Nandra, K., Petrucci, P. O., \& O'Neill, P. M. 2004, MNRAS, 355,929
Malkan, M. A., \& Sargent, W. L. W. 1982, ApJ, 254, 22

Martocchia, A., Karas, V., \& Matt, G. 2000, MNRAS, 312, 817

Matt, G. 2001, X-ray Astronomy: Stellar Endpoints, AGN, and the Diffuse X-ray Background, AIP Conf. Proc., 599, 209

Merloni, A., Malzac, J., Fabian, A. C., \& Ross, R. R. 2006, MNRAS, 370, 1699 Miniutti, G., \& Fabian, A. C. 2004, MNRAS, 349, 1435

Miniutti, G., Fabian, A. C., Goyder, R., \& Lasenby, A. N. 2003, MNRAS, 344, L22

Nandra, K., Turner, T. J., George, I. M., et al. 1995, MNRAS, 273, 85

O'Brien, P. T., Wilson, R., \& Gondhalekar, P. M. 1988, MNRAS, 233, 801

Page, K. L., Schartel, N., Turner, M. J. L., \& O’Brien, P. T. 2004, MNRAS, 352, 523

Pecháček, T., Dovčiak, M., Karas, V., \& Matt, G. 2005, A\&A, 441, 855

Petrucci, P. O., Henri, G., Maraschi, L., et al. 2002, A\&A, 388, L5

Ponti, G., Cappi, M., Dadina, M., \& Malaguti, G. 2004, A\&A, 417, 451

Ponti, G., Miniutti, G., Cappi, M., et al. 2006, MNRAS, 368, 903

Porquet, D. 2006, A\&A, 445, L5

Porquet, D., Reeves, J. N., Uttley, P., \& Turner, T. J. 2004, A\&A, 427, 101

Reeves, J. N., Nandra, K., George, I. M., et al. 2004, ApJ, 602, 648

Ross, R. R., \& Fabian, A. C. 2005, MNRAS, 358, 211

Schurch, N. J., \& Done, C. 2006, MNRAS, 371, 81

Shields, G. A. 1978, Nature, 272, 706

Sobolewska, M. A., \& Done, C. 2007, MNRAS, 374, 150

Tombesi, F., De Marco, B., Iwasawa, K., et al. 2007, [arXiv: 0704.0226]

Turner, T. J., Kraemer, S. B., \& Reeves, J. N. 2004, ApJ, 603, 62

Walter, R., \& Fink, H. H. 1993, A\&A, 274, 105

Zdziarski, A. A., Poutanen, J., \& Johnson, W. N. 2000, ApJ, 542, 703 